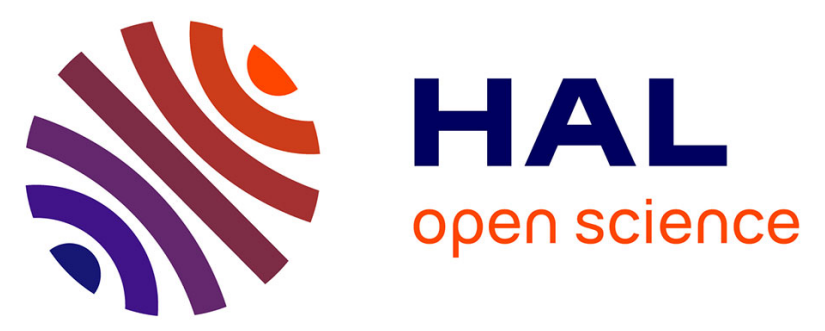

\title{
Full-field vs. homogenization methods to predict microstructure-property relations for polycrystalline materials
}

Ricardo A. Lebensohn, Pedro Ponte Castañeda, Rénald Brenner, Olivier

Castelnau

\section{To cite this version:}

Ricardo A. Lebensohn, Pedro Ponte Castañeda, Rénald Brenner, Olivier Castelnau. Full-field vs. homogenization methods to predict microstructure-property relations for polycrystalline materials. Computational Methods for Microstructure-Property Relationships, Springer, pp.393-441, 2011, 978144190642-7. 10.1007/978-1-4419-0643-4_11. hal-02619028

\section{HAL Id: hal-02619028 https://hal.science/hal-02619028}

Submitted on 25 May 2020

HAL is a multi-disciplinary open access archive for the deposit and dissemination of scientific research documents, whether they are published or not. The documents may come from teaching and research institutions in France or abroad, or from public or private research centers.
L'archive ouverte pluridisciplinaire HAL, est destinée au dépôt et à la diffusion de documents scientifiques de niveau recherche, publiés ou non, émanant des établissements d'enseignement et de recherche français ou étrangers, des laboratoires publics ou privés. 


\title{
Full-Field vs. Homogenization Methods to Predict Microstructure-Property Relations for Polycrystalline Materials
}

\author{
R.A. Lebensohn, P. Ponte Castañeda, R. Brenner, and O. Castelnau
}

\begin{abstract}
In this chapter, we review two recently proposed methodologies, based on crystal plasticity, for the prediction of microstructure-property relations in polycrystalline aggregates. The first, known as the second-order viscoplastic self-consistent (SC) method, is a mean-field theory, while the second, known as the fast Fourier transform (FFT)-based formulation, is a full-field method. The main equations and assumptions underlying both formulations are presented, using a unified notation and pointing out their similarities and differences. Concerning mean-field SC homogenization theories for the prediction of mechanical behavior of nonlinear viscoplastic polycrystals, we carry out detailed comparisons of the different linearization assumptions that can be found in the literature. Then, after validating the FFT-based full-field formulation by comparison with available analytical results, the effective behavior of model material systems predicted by means of different SC approaches are compared with ensemble averages of full-field solutions. These comparisons show that the predictions obtained by means of the secondorder SC approach - which incorporates statistical information at grain level beyond first-order, through the second moments of the local field fluctuations inside the constituent grains - are in better agreement with the FFT-based full-field solutions. This is especially true in the cases of highly heterogeneous materials due to strong nonlinearity or single-crystal anisotropy. The second-order SC approach is next applied to the prediction of texture evolution of polycrystalline ice deformed in compression, a case that illustrates the flexibility of this formulation to handle problems involving materials with highly anisotropic local properties. Finally, a full three-dimensional implementation, the FFT-based formulation, is applied to study subgrain texture evolution in copper deformed in tension, with direct input and validation from orientation images. Measurements and simulations agree in that grains with initial orientation near $<110>$ tend to develop higher misorientations. This behavior
\end{abstract}

\footnotetext{
R.A. Lebensohn (凶)

Materials Science and Technology Division, Los Alamos National Laboratory,

MS G755, Los Alamos, NM 87545, USA

e-mail: lebenso@lanl.gov
} 
can be explained in terms of attraction toward the two stable orientations and grain interaction. Only models like the FFT-based formulation that account explicitly for interaction between individual grains are able to capture these effects.

Keywords Antiplane deformation - Crystal plasticity · Fast Fourier transform - Field fluctuations · Green function method - Mean-field vs. full-field models · Micromechanics · Misorientation - Orientation imaging microscopy formulation · Polycrystal · Second-order homogenization · Texture · Viscoplastic self-consistent

\section{Introduction}

An accurate prediction of the mechanical behavior of polycrystalline aggregates undergoing plastic deformation based on the directional properties and evolving substructure of their constituent single-crystal grains is an indispensable tool to establish the relationship between microstructure and properties of this large and ubiquitous class of materials. On one hand, advances in the theories that link microstructures and properties of nonlinear heterogeneous materials have enabled the development of new concepts and algorithms for the prediction of the effective plastic response of statistically defined classes of polycrystalline aggregates using crystal plasticity-based mean-field approaches. On the other hand, novel and very efficient full-field approaches also based on crystal plasticity have been proposed and applied to the prediction of the actual micromechanical fields that develop inside the grains of polycrystals with particular microstructures. In this chapter, we will review two of the most recent crystal plasticity-based mean-field [i.e., the secondorder (SO) viscoplastic self-consistent (VPSC) theory] and full-field [i.e., the fast Fourier transform (FFT)-based formulation] models, establishing the connections existing between the two formulations, and showing applications of both approaches to the prediction of microstructure-property relations of polycrystalline aggregates.

Concerning mean-field approximations, the computation of effective mechanical response and texture evolution of polycrystalline materials using homogenization approaches has a long tradition (Sachs 1928; Taylor 1938). At present, self-consistent approximations are extensively used to deal with this problem. The 1-site viscoplastic (VP) self-consistent (SC) theory of polycrystal deformation can be traced back to the seminal work of Molinari et al. (1987). Later, Lebensohn and Tomé (1993) implemented this formulation numerically to fully account for polycrystal anisotropy, developing the first version of the VPSC code. In the last decade, this code has experienced several improvements and extensions (Tomé and Lebensohn 2008), and it is nowadays extensively used to simulate plastic deformation of polycrystalline aggregates and to interpret experimental evidence on metallic, geological, and polymeric materials (see Lebensohn et al. 2007 for a comprehensive list of material systems studied with the VPSC theory and code). 
The self-consistent approximation, one of the most commonly used homogenization methods to estimate the mechanical response behavior of polycrystals, was originally proposed by Hershey (1954) for linear elastic materials. For nonlinear aggregates (as those formed by grains deforming in the viscoplastic regime), the several self-consistent approximations that were proposed subsequently differ in the procedure used to linearize the nonlinear local mechanical behavior, but eventually all of them end up making use of the original linear self-consistent theory. Among the nonlinear SC formulations, we can mention: the secant (SEC) (Hill 1965; Hutchinson 1976), the tangent (TG) (Molinari et al. 1987; Lebensohn and Tomé 1993), and the affine (AFF) (Ponte Castañeda 1996; Masson et al. 2000) approximations. All these are first-order SC approximations since they are based on linearization schemes that, at grain level, make use of information on field averages only, disregarding higher-order statistical information inside the grains. However, the above assumption may be questionable specially when strong directionality and/or large variations in local properties are to be expected. Such is the case for low rate-sensitivity materials, aggregates made of highly anisotropic grains, and multiphase polycrystals. In all those cases, strong deformation gradients are likely to develop inside grains because of the contrast in properties between neighboring grains.

To overcome the above limitations, Ponte Castañeda and coauthors have developed over the last two decades more accurate nonlinear homogenization methods, using linearization schemes at grain level that also incorporate information on the second moments of the field fluctuations in the grains. These more elaborate SC formulations are based on the use of so-called linear comparison methods, which express the effective potential of the nonlinear VP polycrystal in terms of that of a linearly viscous aggregate with properties that are determined from suitably designed variational principles. Ponte Castañeda's first variational method was originally proposed for nonlinear composites (Ponte Castañeda 1991) and then extended to VP polycrystals (deBotton and Ponte Castañeda 1995). It makes use of the SC approximation for linearly viscous polycrystals to obtain bounds and estimates for nonlinear VP polycrystals. The most recent second-order method, proposed for nonlinear composites (Ponte Castañeda 2002), and later extended to VP polycrystals (Liu and Ponte Castañeda 2004), uses the SC approximation for a more general class of linearly viscous polycrystals, having a non-vanishing strain-rate at zero stress, to generate even more accurate SC estimates for VP polycrystals. The implementation of a fully anisotropic second-order approach inside the VPSC code has been a necessary step toward improving its predictive capability for polycrystalline materials that exhibit high contrast in local properties. Unavoidably, this improved capability comes at the expense of more complex and numerically demanding algorithms.

In what concerns full-field approaches, in the last 20 years, crystal plasticitybased finite element (FE) implementations have been extensively applied to obtain solutions for the plastic deformation of polycrystalline materials with intracrystalline resolution (Becker 1991; Mika and Dawson 1998; Delaire et al. 2001; Barbe et al. 2001; Raabe et al. 2001; Bhattacharyya et al. 2001; Delannay et al. 2003, 2006; Cheong and Busso 2004; Diard et al. 2005; Musienko et al. 2007). However, the large number of degrees of freedom required by such FE calculations limits the size of the microstructures that can be investigated by these methods. Conceived as a 
very efficient alternative to FE methods, a formulation inspired by image-processing techniques and based on the FFT algorithm, originally proposed by Moulinec and Suquet (1994), for the prediction of the micromechanical behavior of plastically deforming heterogeneous materials. The latter includes both composites (Moulinec and Suquet 1998; Michel et al. 2000; Idiart et al. 2006), in which the source of heterogeneity is related to the spatial distribution of phases with different mechanical properties, and polycrystals (Lebensohn 2001; Lebensohn et al. 2004a, b, 2005, 2008), in which the heterogeneity is related to the spatial distribution of crystals with directional mechanical properties.

The plan of this chapter is as follows. In Sect. 2, we describe the implementation of the second-order formulation inside the VPSC code (Lebensohn et al. 2007), and present the FFT-based formulation, specialized to the case of viscoplastic polycrystals (Lebensohn et al. 2008). In Sect. 3, we first show a validation of the FFT-based approach by comparison with an exact analytical result and then discuss the differences between the first- and second-order VPSC formulations by comparing their predictions with corresponding FFT-based full-field solutions. We do so for crystals with different symmetries, as a function of anisotropy, number of independent slip systems, and degree of nonlinearity. In this comparison, the second-order estimates show the best overall agreement with the full-field solutions. The different SC approaches are then applied to the prediction of texture evolution in a strongly heterogeneous system (i.e., polycrystalline ice deforming in uniaxial compression) (Lebensohn et al. 2007). This comparison shows that the second-order formulation yields results in better agreement with experimental evidence than the first-order approximations, predicting a substantial and persistent accommodation of deformation by basal slip, even when the basal poles become strongly aligned with the compression direction. Section 3 also shows an application of the FFT-based formulation to the prediction of subgrain texture and microstructure evolution in polycrystalline copper deformed under tension, with direct input from orientation imaging microscopy (OIM) images (Lebensohn et al. 2008). Average orientations and misorientations predicted after $11 \%$ tensile strain are directly compared with OIM measurements. Experiments and simulations agree in that grains with initial orientation near $<110>$ tend to develop higher misorientations. This behavior can be explained in terms of attraction toward the two stable orientations and grain interaction. Only models that account explicitly for interaction between individual grains, like the FFT-based formulation, are able to capture these effects.

\section{Models}

\subsection{Viscoplastic Self-Consistent Formalism}

In this section, the incompressible viscoplastic self-consistent formulation (Lebensohn et al. 1998) is first presented using the affine linearization scheme (Ponte Castañeda 1996; Masson et al. 2000), and the second-order linearization procedure (Ponte Castañeda 2002; Liu and Ponte Castañeda 2004) is described next. 
The self-consistent formulation consists in representing a polycrystal by means of weighted, ellipsoidal, statistically representative (SR) grains. Each of these SR grains represents the average behavior of all the grains with a particular crystallographic orientation and morphology, but different environments. These SR grains should be regarded as representing the behavior of mechanical phases, i.e., all the single crystals with a given orientation $(r)$ belong to mechanical phase $(r)$ and are represented by SR grain $(r)$. (Note the difference between "mechanical phases," which differ from each other only in terms of crystallographic orientation and/or morphology, and actual "phases" differing from each other in crystallographic structure and/or composition). In what follows, "SR grain $(r)$ " and "mechanical phase $(r)$ " will be used interchangeably. The weights represent volume fractions. The latter are chosen to reproduce the initial texture of the material. In turn, each representative grain will be treated as an ellipsoidal viscoplastic inclusion embedded in an effective viscoplastic medium. Both inclusion and medium have fully anisotropic properties. Deformation is carried by crystal plasticity mechanisms: slip and twinning systems activated by a resolved shear stress.

\subsubsection{Local Constitutive Behavior and Homogenization}

Let us consider a macroscopic velocity-gradient $V_{i, j}$ applied to an polycrystalline aggregate, which can be decomposed into an average symmetric strain-rate $\dot{E}_{i j}=$ $\frac{1}{2}\left(V_{i, j}+V_{j, i}\right)$ and an average antisymmetric rotation-rate $\dot{\Omega}_{i j}=\frac{1}{2}\left(V_{i, j}-V_{j, i}\right)$. Let us assume that the plastic component of the deformation is much larger than the elastic part and therefore the flow is incompressible. The viscoplastic constitutive behavior at each material point $\mathbf{x}$ (in what follows, Cartesian and Fourier vectors are indicated in boldface, second and fourth-rank tensors, either in components or not, are not) is described by means of the following nonlinear, rate-sensitive equation:

$\left.\dot{\varepsilon}(\mathbf{x})=\sum_{k=1}^{N_{k}} m^{k}(\mathbf{x}) \dot{\gamma}^{k}(\mathbf{x})=\dot{\gamma}_{\mathrm{o}} \sum_{k=1}^{N_{k}} m^{k}(\mathbf{x}) \frac{\left|m^{k}(\mathbf{x}): \sigma^{\prime}(\mathbf{x})\right|}{\tau_{\mathrm{o}}^{k}(\mathbf{x})}\right)^{n} \times \operatorname{sgn}\left(m^{k}(\mathbf{x}): \sigma^{\prime}(\mathbf{x})\right)$,

where the symbol ":" indicates double contraction of indices, and the sum runs over all $N_{k}$ slip and twinning systems. $\tau_{\mathrm{o}}^{k}$ and $m^{k}(\mathbf{x})=\frac{1}{2}\left(n^{k}(\mathbf{x}) \otimes b^{k}(\mathbf{x})+b^{k}(\mathbf{x}) \otimes\right.$ $\left.n^{k}(\mathbf{x})\right)$ are the threshold resolved shear stress and the symmetric Schmid tensor associated with slip or twinning system $(k)$ (with $\mathbf{n}^{k}$ and $\mathbf{b}^{k}$ being the normal and Burgers vector direction of such slip or twinning system), $\dot{\varepsilon}$ and $\sigma^{\prime}$ are the deviatoric strain-rate and stress tensors, $\dot{\gamma}^{k}$ is the local shear-rate on slip or twinning system $(k), \dot{\gamma}_{\mathrm{o}}$ is a normalization factor, and $n$ is the rate-sensitivity exponent. Note that although (1) can be used to deal with crystal deforming by slip and twinning, in the examples that follows (in the context of both homogenization and full-field approaches), we will only consider crystal deformation by slip. In this way, we avoid the additional complication of having to deal with twinning reorientation. 
Also note that the constitutive behavior described in (1) does not consider other high temperature crystal deformation mechanisms, such as climb, grain-boundary sliding, or recrystallization, and that elastic effects are neglected.

For later use, the plastic rotation-rate associated with a material point $\mathbf{x}$ contributing to the crystallographic lattice rotation is given by:

$$
\dot{\omega}_{i j}^{p}(\mathbf{x})=\sum_{k} \alpha_{i j}^{k}(\mathbf{x}) \dot{\gamma}^{k}(\mathbf{x}),
$$

where $\alpha^{s}(\mathbf{x})=\frac{1}{2}\left(n^{s}(\mathbf{x}) \otimes b^{s}(\mathbf{x})-b^{s}(\mathbf{x}) \otimes n^{s}(\mathbf{x})\right)$ is the antisymmetric Schmid tensor.

Let us assume that the following linear relation [i.e., an approximation of the actual nonlinear relation (1)] holds between the strain-rate and stress in the SR grain $(r)$ :

$$
\dot{\varepsilon}(\mathbf{x})=M^{(r)}: \sigma^{\prime}(\mathbf{x})+\dot{\varepsilon}^{o(r)},
$$

where $M^{(r)}$ and $\dot{\varepsilon}^{o(r)}$ are, respectively, the viscoplastic compliance and the backextrapolated term of SR grain $(r)$. Depending on the linearization assumption, $M^{(r)}$ and $\dot{\varepsilon}^{o(r)}$ can be chosen differently (some possible choices are discussed below). Taking a volumetric average, we obtain:

$$
\dot{\varepsilon}^{(r)}=M^{(r)}: \sigma^{(r)}+\dot{\varepsilon}^{o(r)},
$$

where $\dot{\varepsilon}^{(r)}$ and $\sigma^{\prime(r)}$ are average magnitudes in the volume of SR grain $(r)$. Let us homogenize the behavior of a linear heterogeneous medium whose local behavior is described in (3) assuming an analogous linear relation at the effective medium (macroscopic) level:

$$
\dot{E}=\bar{M}: \Sigma^{\prime}+\dot{E}^{o},
$$

where $\dot{E}$ and $\Sigma^{\prime}$ are the overall (macroscopic) deviatoric strain-rate and stress tensors and $\bar{M}$ and $\dot{E}^{o}$ are, respectively, the viscoplastic compliance and backextrapolated term of an a priori unknown homogeneous equivalent medium (HEM). The usual procedure to obtain the homogenized response of a linear polycrystal is the linear self-consistent method. The problem underlying the self-consistent method is that of an inhomogeneous domain $(r)$ of moduli $M^{(r)}$ and $\dot{\varepsilon}^{o(r)}$, embedded in an infinite medium of moduli $\bar{M}$ and $\dot{E}^{o}$. Invoking the concept of the equivalent inclusion (Mura 1987), the local constitutive behavior in domain $(r)$ can be rewritten as:

$$
\dot{\varepsilon}(\mathbf{x})=\bar{M}: \sigma^{\prime}(\mathbf{x})+\dot{E}^{o}+\dot{\varepsilon}^{*}(\mathbf{x}),
$$

where $\dot{\varepsilon}^{*}(\mathbf{x})$ is an eigen-strain-rate field, which follows from replacing the inhomogeneity by an equivalent inclusion. Rearranging and subtracting (5) from (6) gives:

$$
\tilde{\sigma}^{\prime}(\mathbf{x})=\bar{L}:\left(\tilde{\dot{\varepsilon}}(\mathbf{x})-\dot{\varepsilon}^{*}(\mathbf{x})\right) .
$$


The symbol " " denotes local deviations from macroscopic values of the corresponding magnitudes, and $\bar{L}=\bar{M}^{-1}$. Combining (7) with the equilibrium condition gives:

$$
\sigma_{i j, j}(\mathbf{x})=\tilde{\sigma}_{i j, j}(\mathbf{x})=\tilde{\sigma}_{i j, j}^{\prime}(\mathbf{x})+\tilde{\sigma}_{, i}^{m}(\mathbf{x}),
$$

where $\sigma_{i j}$ and $\sigma^{m}$ are the Cauchy stress tensor and the mean stress, respectively. Using the relation $\tilde{\dot{\varepsilon}}_{i j}(\mathbf{x})=\frac{1}{2}\left(\tilde{v}_{i, j}(\mathbf{x})+\tilde{v}_{j, i}(\mathbf{x})\right)$ between the strain-rate and velocity-gradient deviations, and adding the incompressibility condition associated with plastic deformation, we obtain:

$$
\mid \begin{aligned}
& \bar{L}_{i j k l} \tilde{v}_{k, l j}(\mathbf{x})+\tilde{\sigma}_{, i}^{m}(\mathbf{x})+\varphi_{i j, j}(\mathbf{x})=0 \\
& \tilde{v}_{k, k}(\mathbf{x})=0
\end{aligned},
$$

where

$$
\varphi_{i j}(\mathbf{x})=-\bar{L}_{i j k l} \dot{\varepsilon}_{k l}^{*}(\mathbf{x})
$$

is a heterogeneity or polarization field, and its divergence: $f_{i}(\mathbf{x})=\varphi_{i j, j}(\mathbf{x})$ is a fictitious volumetric force field. System (9) consists of four differential equations with four unknowns: three are the components of velocity deviation vector $\tilde{v}_{i}(\mathbf{x})$, and one is the mean stress deviation $\tilde{\sigma}^{m}(\mathbf{x})$. A system of $N$ linear differential equations with $N$ unknown functions and a polarization term can be solved using the Green function method. Let us call $G_{k m}(\mathbf{x})$ and $H_{m}(\mathbf{x})$ the Green functions associated with $\tilde{v}_{i}(\mathbf{x})$ and $\tilde{\sigma}^{m}(\mathbf{x})$, respectively, which solve the auxiliary problem of a unitary volumetric force, with a single non-vanishing $m$-component:

$$
\mid \begin{aligned}
& \bar{L}_{i j k l} G_{k m, l j}\left(\mathbf{x}-\mathbf{x}^{\prime}\right)+H_{m, i}\left(\mathbf{x}-\mathbf{x}^{\prime}\right)+\delta_{i m} \delta\left(\mathbf{x}-\mathbf{x}^{\prime}\right)=0, \\
& G_{k m, k}\left(\mathbf{x}-\mathbf{x}^{\prime}\right)=0
\end{aligned}
$$

Once the solution of (11) is obtained, the solution for the velocity field is given by the convolution integral:

$$
\tilde{v}_{k}(\mathbf{x})=\int_{R^{3}} G_{k i}\left(\mathbf{x}-\mathbf{x}^{\prime}\right) f_{i}\left(\mathbf{x}^{\prime}\right) \mathrm{d} x^{\prime} .
$$

System (11) can be solved using the Fourier transform method. Expressing the Green functions in terms of their inverse Fourier transforms, the differential system (11) can be transformed into an algebraic system:

$$
\mid \begin{aligned}
& \alpha_{j} \alpha_{l} \bar{L}_{i j k l} k^{2} \hat{G}_{k m}(\xi)+\alpha_{i} i k \hat{H}_{m}(\xi)=\delta_{i m}, \\
& \alpha_{k} k^{2} \hat{G}_{k m}(\xi)=0,
\end{aligned}
$$

where $k$ and $\alpha$ are the modulus and the unit vector associated with a point of Fourier space $\xi=k \alpha$, respectively. Calling $A_{i k}^{\prime}(\alpha)=\alpha_{j} \alpha_{l} \bar{L}_{i j k l}$, system (13) can be 
expressed as a matrix product $\mathbf{A} \times \mathbf{B}=\mathbf{C}$, w h e r e $\mathbf{A}, \mathbf{B}$, a n d $\mathbf{C}$ are the matrices given by:

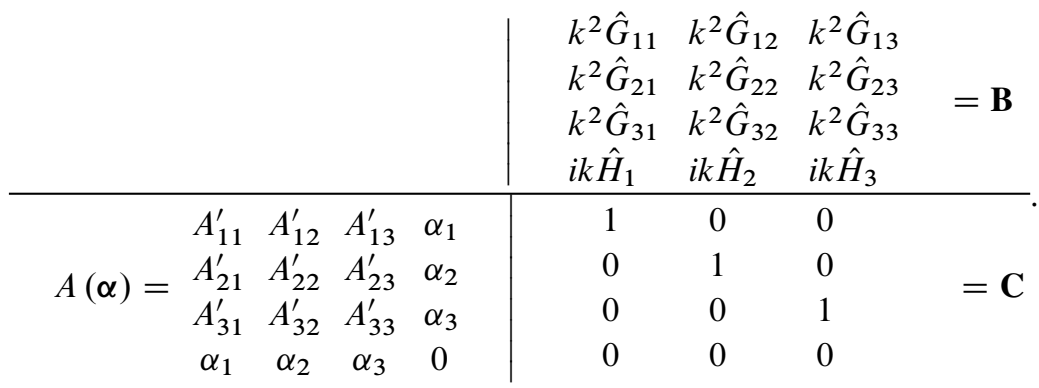

Using the explicit form of matrix $\mathbf{C}$, we can write:

$$
\mathbf{B}=\mathbf{A}^{-1} \times \mathbf{C}=\left[\begin{array}{ccc}
A_{11}^{-1} & A_{12}^{-1} & A_{13}^{-1} \\
A_{21}^{-1} & A_{22}^{-1} & A_{23}^{-1} \\
A_{31}^{-1} & A_{32}^{-1} & A_{33}^{-1} \\
A_{41}^{-1} & A_{42}^{-1} & A_{43}^{-1}
\end{array}\right]
$$

Finally, comparing (14) and (15):

$$
k^{2} \hat{G}_{i j}(\xi)=A_{i j}^{-1}(\alpha)(i, j=1,3) .
$$

Since the components of $\mathbf{A}$ are real functions of $\alpha$, so are those of $k^{2} \hat{G}_{i j}(\xi)$. This property leads to real integrals in the derivation that follows.

Knowing the Green tensor expression in Fourier space, we can write the solution of our eigen-strain-rate problem using the convolution integral. Taking partial derivatives to (12), we obtain:

$$
\tilde{v}_{k, l}(\mathbf{x})=\int_{R^{3}} G_{k i, l}\left(\mathbf{x}-\mathbf{x}^{\prime}\right) f_{i}\left(\mathbf{x}^{\prime}\right) \mathrm{d} \mathbf{x}^{\prime}
$$

Replacing the expression of the fictitious volumetric force field in (17), recalling that $\partial G_{i j}\left(\mathbf{x}-\mathbf{x}^{\prime}\right) / \partial \mathbf{x}=-\partial G_{i j}\left(\mathbf{x}-\mathbf{x}^{\prime}\right) / \partial \mathbf{x}^{\prime}$, integrating by parts, and using the divergence theorem (Mura 1987), we obtain:

$$
\tilde{v}_{k, l}(\mathbf{x})=\int_{R^{3}} G_{k i, j l}\left(\mathbf{x}-\mathbf{x}^{\prime}\right) \varphi_{i j}\left(\mathbf{x}^{\prime}\right) \mathrm{d} \mathbf{x}^{\prime} .
$$

The integral equation (18) provides an exact implicit solution to the problem. Furthermore, it is known from Eshelby's elastic inclusion formalism that if the eigen-strain is uniform over an ellipsoidal domain where the stiffness tensor is uniform, then the stress and the strain are constant over the domain of the inclusion $(r)$. The latter suggests to use an a priori unknown constant polarization within the 
volume $\Omega$ of the ellipsoidal inclusion. This allows us to average the local field (18) over the domain $\Omega$ and obtain an average strain-rate inside the inclusion of the form:

$$
\tilde{v}_{k, l}^{(r)}=\left(-\frac{1}{\Omega} \int_{\Omega} \int_{\Omega} G_{k i, j l}\left(\mathbf{x}-\mathbf{x}^{\prime}\right) \mathrm{d} \mathbf{x} \mathrm{d} \mathbf{x}^{\prime}\right) \bar{L}_{i j m n} \dot{\varepsilon}_{m n}^{*(r)},
$$

where $\tilde{v}_{k, l}^{(r)}$ and $\dot{\varepsilon}_{m n}^{*(r)}$ have to be interpreted as average quantities inside the inclusion. Expressing the Green tensor in terms of the inverse Fourier transform and taking derivatives, we obtain:

$$
\begin{aligned}
\tilde{v}_{k, l}^{(r)} & =\left(\frac{1}{8 \pi^{3} \Omega} \int_{\Omega} \int_{\Omega} \int_{R^{3}} \alpha_{j} \alpha_{l}\left(k^{2} \hat{G}_{k i}(\boldsymbol{\xi})\right) \exp \left[-i \xi\left(\mathbf{x}-\mathbf{x}^{\prime}\right)\right] \mathrm{d} \boldsymbol{\xi} \mathrm{d} \mathbf{x d} \mathbf{x}^{\prime}\right) \bar{L}_{i j m n} \dot{\varepsilon}_{m n}^{*(r)} \\
& =T_{k l i j} \bar{L}_{i j m n} \dot{\varepsilon}_{m n}^{*(r)} .
\end{aligned}
$$

Writing $d \xi$ in spherical coordinates: $d \xi=k^{2} \sin \theta \mathrm{d} k \mathrm{~d} \theta \mathrm{d} \varphi$ and using relation (16), the Green interaction tensor $T_{k l i j}$ can be expressed as:

$$
T_{k l i j}=\frac{1}{8 \pi^{3} \Omega} \int_{0}^{2 \pi} \int_{0}^{\pi} \alpha_{j} \alpha_{l} A_{k i}^{-1}(\alpha) \Lambda(\alpha) \sin \theta \mathrm{d} \theta \mathrm{d} \varphi,
$$

where

$$
\Lambda(\alpha)=\int_{0}^{\infty}\left(\int_{\Omega} \int_{\Omega} \exp \left[-i \xi\left(\mathbf{x}-\mathbf{x}^{\prime}\right)\right] d \mathbf{x} d \mathbf{x}^{\prime}\right) k^{2} \mathrm{~d} k .
$$

Integrating (22) inside an ellipsoidal grain of radii $(a, b, c)$ (Berveiller et al. 1987) and replacing in (21) gives:

$$
T_{k l i j}=\frac{a b c}{4 \pi} \int_{0}^{2 \pi} \int_{0}^{\pi} \frac{\alpha_{j} \alpha_{l} A_{k i}^{-1}(\alpha)}{[\rho(\alpha)]^{3}} \sin \theta \mathrm{d} \theta \mathrm{d} \varphi,
$$

where $\rho(\alpha)=\left[\left(a \alpha_{1}\right)^{2}+\left(b \alpha_{2}\right)^{2}+\left(c \alpha_{3}\right)^{2}\right]^{1 / 2}$. The symmetric and antisymmetric Eshelby tensors (functions of $\bar{L}$ and the shape of the ellipsoidal inclusion, representing the morphology of the SR grains) are defined as:

$$
\begin{gathered}
S_{i j k l}=\frac{1}{4}\left(T_{i j m n}+T_{j i m n}+T_{i j n m}+T_{j i n m}\right) \bar{L}_{m n k l}, \\
\Pi_{i j k l}=\frac{1}{4}\left(T_{i j m n}-T_{j i m n}+T_{i j n m}-T_{j i n m}\right) \bar{L}_{m n k l} .
\end{gathered}
$$

Taking symmetric and antisymmetric components to (20) and using (24) and (25), we obtain the average strain-rate and rotation-rate deviations of the ellipsoidal domain:

$$
\begin{aligned}
\tilde{\dot{\varepsilon}}^{(r)} & =S: \dot{\varepsilon}^{*(r)}, \\
\tilde{\dot{\omega}}^{(r)} & =\Pi: \dot{\varepsilon}^{*(r)}=\Pi: S^{-1}: \tilde{\dot{\varepsilon}}^{(r)},
\end{aligned}
$$


where $\tilde{\varepsilon}^{(r)}=\dot{E}-\dot{\varepsilon}^{(r)}$ and $\tilde{\dot{\omega}}^{(r)}=\dot{\Omega}-\dot{\omega}^{(r)}$ are deviations of the average strain-rate and rotation-rate inside the inclusion, with respect to the corresponding overall magnitudes, and $\dot{\varepsilon}^{*(r)}$ is the average eigen-strain-rate in the inclusion.

\subsubsection{Interaction and Localization Equations}

Taking volume averages over the domain of the inclusion on both sides of (7) gives:

$$
\tilde{\sigma}^{\prime(r)}=\bar{L}:\left(\tilde{\dot{\varepsilon}}^{(r)}-\dot{\varepsilon}^{*(r)}\right) .
$$

Replacing the eigen-strain-rate given by (26) into (28), we obtain the interaction equation:

$$
\tilde{\dot{\varepsilon}}^{(r)}=-\tilde{M}: \tilde{\sigma}^{\prime(r)},
$$

where the interaction tensor is given by:

$$
\tilde{M}=(I-S)^{-1}: S: \bar{M} .
$$

Replacing the constitutive relations of the inclusion and the effective medium in the interaction equation and after some manipulation, one can write the following localization equation:

$$
\sigma^{(r)}=B^{(r)}: \Sigma^{\prime}+b^{(r)},
$$

where the localization tensors are defined as:

$$
\begin{aligned}
& B^{(r)}=\left(M^{(r)}+\tilde{M}\right)^{-1}:(\bar{M}+\tilde{M}), \\
& b^{(r)}=\left(M^{(r)}+\tilde{M}\right)^{-1}:\left(\dot{E}^{o}-\dot{\varepsilon}^{o(r)}\right) .
\end{aligned}
$$

\subsubsection{Self-Consistent Equations}

The derivation presented in the previous sections solves the problem of an equivalent inclusion embedded in an effective medium. In this section, we use the previous result to construct a polycrystal model, consisting in regarding each SR grain $(r)$ as an inclusion embedded in an effective medium that represents the polycrystal. The properties of such medium are not known a priori but have to be found through an iterative procedure. Replacing the stress localization equation (31) in the average local constitutive equation (4), we obtain:

$$
\varepsilon^{(r)}=M^{(r)}: B^{(r)}: \Sigma+M^{(r)}: b^{(r)}+\varepsilon^{o(r)} .
$$


Taking volumetric average to (34), enforcing the condition that the average of the strain-rates over the aggregate has to coincide with the macroscopic quantities, i.e.:

$$
\dot{E}=\left\langle\dot{\varepsilon}^{(r)}\right\rangle,
$$

where the brackets " (\rangle " denote average over the SR grains, weighted by the associated volume fraction, and using the macroscopic constitutive relation (5), we obtain the following self-consistent equations for the HEM's compliance and backextrapolated term:

$$
\begin{aligned}
\bar{M} & =\left\langle M^{(r)}: B^{(r)}\right\rangle, \\
\dot{E}^{o} & =\left\langle M^{(r)}: b^{(r)}+\dot{\varepsilon}^{o(r)}\right\rangle .
\end{aligned}
$$

These self-consistent equations are derived imposing the average of the local strainrates to coincide with the applied macroscopic strain-rate (35). If all the SR grains are represented by ellipsoids that have the same shape and orientation, it can be shown that the same equations are obtained from the condition that the average of the local stresses coincides with the macroscopic stress. If the SR grains have different morphologies, they have associated different Eshelby tensors, and the interaction tensors cannot be factored from the averages. In such case, the following generalized self-consistent expressions should be used (Walpole 1969):

$$
\begin{aligned}
& \bar{M}=\left\langle M^{(r)}: B^{(r)}\right\rangle:\left\langle B^{(r)}\right\rangle^{-1}, \\
& \dot{E}^{o}=\left\langle M^{(r)}: b^{(r)}+\dot{\varepsilon}^{o(r)}\right\rangle-\left\langle M^{(r)}: B^{(r)}\right\rangle:\left\langle B^{(r)}\right\rangle^{-1}:\left\langle b^{(r)}\right\rangle .
\end{aligned}
$$

\subsubsection{Linearization Assumptions}

As stated earlier, different choices are possible for the linearized behavior at grain level, and the results of the homogenization scheme depend on this choice. In what follows, we present several first-order linearization schemes, defined in terms of the stress first-order moment (average) inside SR grain $(r)$.

The secant approximation (Hill 1965; Hutchinson 1976) consists in assuming the following linearized moduli:

$$
\begin{aligned}
& \left.M_{\mathrm{sec}}^{(r)}=\dot{\gamma}_{\mathrm{o}} \sum_{k} \frac{m^{k(r)} \otimes m^{k(r)}}{\tau_{\mathrm{o}}^{k(r)}} \frac{m^{k(r)}: \sigma^{\prime(r)}}{\tau_{\mathrm{o}}^{k(r)}}\right)^{n-1}, \\
& \dot{\varepsilon}_{\mathrm{sec}}^{o(r)}=0
\end{aligned}
$$


where the index $(r)$ in $m^{k(r)}$ and $\tau_{\mathrm{o}}^{k(r)}$ indicates uniform (average) values of these magnitudes, corresponding to a given orientation and hardening state associated with SR grain $(r)$.

Under the affine approximation (Ponte Castañeda 1996; Masson et al. 2000), the moduli are given by:

$$
\begin{aligned}
& \left.M_{\mathrm{aff}}^{(r)}=n \dot{\gamma}_{\mathrm{o}} \sum_{k} \frac{m^{k(r)} \otimes m^{k(r)}}{\tau_{\mathrm{o}}^{k(r)}} \frac{m^{k(r)}: \sigma^{\prime(r)}}{\tau_{\mathrm{o}}^{k(r)}}\right)^{n-1}, \\
& \left.\dot{\varepsilon}_{\mathrm{aff}}^{o(r)}=(1-n) \dot{\gamma}_{\mathrm{o}} \sum_{k} \frac{m^{k(r)}: \sigma^{\prime(r)}}{\tau_{\mathrm{o}}^{k(r)}}\right)^{n} \times \operatorname{sgn}\left(m^{k(r)}: \sigma^{\prime(r)}\right) .
\end{aligned}
$$

In the case of the tangent approximation (Molinari et al. 1987; Lebensohn and Tomé 1993), the moduli are, formally, the same as in the affine case: $M_{\mathrm{tg}}^{(r)}=M_{\mathrm{aff}}^{(r)}$ and $\dot{\varepsilon}_{\mathrm{tg}}^{o(r)}=\dot{\varepsilon}_{\mathrm{aff}}^{o(r)}$. However, instead of using these moduli, and to avoid the iterative adjustment of the macroscopic back-extrapolated term, Molinari et al. (1987) used the secant SC compliance (38) to adjust $\bar{M}$ (to be denoted $\bar{M}_{\text {sec }}$ ), in combination with the tangent-secant relation: $\bar{M}_{\mathrm{tg}}=n \bar{M}_{\mathrm{sec}}$ (Hutchinson 1976). Then, the expression of the interaction tensor is given by:

$$
\tilde{M}=(I-S)^{-1}: S: \bar{M}_{\mathrm{tg}}=n(I-S)^{-1}: S: \bar{M}_{\mathrm{sec}}
$$

Qualitatively, the interaction equation (29) indicates that the larger the interaction tensor, the smaller the deviation of grain stresses with respect to the average stress should be. As a consequence, for $n \rightarrow \infty$, the tangent approximation tends to a uniform stress state [Sachs (1928) or lower-bound approximation]. This rate-insensitive limit of the tangent formulation is an artifact created using the above tangent-secant relation of the nonlinear polycrystal in the self-consistent solution of the linear comparison polycrystal. On the other hand, the secant interaction has been proven to tend to a uniform strain-rate state [Taylor (1938) or upper-bound approximation] in the rate-insensitive limit.

\subsubsection{Second-Order Formulation}

The more sophisticated second-order approximation to linearize the behavior of the mechanical phases is based on the calculation of average fluctuations of the stress distribution inside the linearized SR grains. The methodology to obtain these fluctuations were derived by Bobeth and Diener (1987), Kreher (1990), and Parton and Buryachenko (1990), and reads as follows. The effective stress potential $\bar{U}_{T}$ of a linearly viscous polycrystal described by (5) may be written in the form (Laws 1973; Willis 1981):

$$
\bar{U}_{T}=\frac{1}{2} \bar{M}::\left(\Sigma^{\prime} \otimes \Sigma^{\prime}\right)+\dot{E}^{o}: \Sigma^{\prime}+\frac{1}{2} \bar{G},
$$


where $\bar{G}$ is the power under zero applied stress. Let us rewrite the self-consistent expression for $\bar{M}$ and $\dot{E}^{o}(36)$ as:

$$
\begin{gathered}
\bar{M}=\left\langle M^{(r)}: B^{(r)}\right\rangle=\sum_{r} c^{(r)} M^{(r)}: B^{(r)}, \\
\dot{E}^{o}=\left\langle M^{(r)}: b^{(r)}+\dot{\varepsilon}^{o(r)}\right\rangle=\sum_{r} c^{(r)}\left(M^{(r)}: b^{(r)}+\dot{\varepsilon}^{o(r)}\right)=\sum_{r} c^{(r)} \dot{\varepsilon}^{o(r)}: B^{(r)},
\end{gathered}
$$

where $c^{(r)}$ is the volume fraction associated with SR grain $(r)$. The corresponding expression for $\bar{G}$ is:

$$
\bar{G}=\sum_{r} c^{(r)} \dot{\varepsilon}^{o(r)}: b^{(r)}
$$

The average second-order moment of the stress field over a SR grain $(r)$ of this polycrystal is a fourth-rank tensor given by:

$$
\left\langle\sigma^{\prime} \otimes \sigma^{\prime}\right\rangle^{(r)}=\frac{2}{c^{(r)}} \frac{\partial \bar{U}_{T}}{\partial M^{(r)}}
$$

Replacing (44-46) in (47), we obtain:

$$
\left\langle\sigma^{\prime} \otimes \sigma^{\prime}\right\rangle^{(r)}=\frac{1}{c^{(r)}} \frac{\partial \bar{M}}{\partial M^{(r)}}::\left(\Sigma^{\prime} \otimes \Sigma^{\prime}\right)+\frac{1}{c^{(r)}} \frac{\partial \dot{E}^{o}}{\partial M^{(r)}}: \Sigma^{\prime}+\frac{1}{c^{(r)}} \frac{\partial \bar{G}}{\partial M^{(r)}} .
$$

Using matrix notation for symmetric deviatoric tensors (Lequeu et al. 1987), the first derivative in the right term can be obtained solving the following equation:

$$
\Omega_{i j k l} \frac{\partial \bar{M}_{k l}}{\partial M_{u \nu}^{(r)}}=\pi_{i j}^{(r, u v)}
$$

where $i, j, k, l$ and $u, v=1,5$. The expressions for $\Omega_{i j k l}$ and $\pi_{i j}^{(r, u v)}$ are given in the Appendix. Expression (49) is a linear system of 25 equations with 25 unknowns (i.e., the components of $\left.\partial \bar{M}_{k l} / \partial M_{u v}^{(r)}\right)$. In turn, the other two derivatives appearing in (48) can be calculated as:

$$
\begin{aligned}
\frac{\partial E_{i}^{o}}{\partial M_{u \nu}^{(r)}} & =\zeta_{i k l} \frac{\partial \bar{M}_{k l}}{\partial M_{u \nu}^{(r)}}+\kappa_{i}^{(r, u v)}, \\
\frac{\partial \bar{G}}{\partial M_{u \nu}^{(r)}} & =\varphi_{i j} \frac{\partial \bar{M}_{i j}}{\partial M_{u \nu}^{(r)}}+\vartheta_{i} \frac{\partial E_{i}^{o}}{\partial M_{u \nu}^{(r)}}+\eta^{(r, u v)},
\end{aligned}
$$

where $\zeta_{i k l}, \varphi_{i j}, \vartheta_{i}, \kappa_{i}^{(r, u v)}$ and $\eta^{(r, u v)}$ are given in Appendix. 
Once the average second moments of the stress are obtained, the corresponding second moments of the strain-rate can be calculated as:

$\langle\dot{\varepsilon} \otimes \dot{\varepsilon}\rangle^{(r)}=\left(M^{(r)} \otimes M^{(r)}\right)::\left\langle\sigma^{\prime} \otimes \sigma^{\prime}\right\rangle^{(r)}+\dot{\varepsilon}^{(r)} \otimes \dot{\varepsilon}^{o(r)}+\dot{\varepsilon}^{o(r)} \otimes \dot{\varepsilon}^{(r)}-\dot{\varepsilon}^{o(r)} \otimes \dot{\varepsilon}^{o(r)}$.

The average second moments can be used, for instance, to generate the average second moment of the equivalent stress and strain-rate in mechanical phase $(r)$ as:

$$
\begin{aligned}
& \overline{\bar{\sigma}}_{\mathrm{eq}}^{(r)}=\left(\frac{3}{2} I::\left\langle\sigma^{\prime} \otimes \sigma^{\prime}\right\rangle^{(r)}\right)^{1 / 2}, \\
& \overline{\dot{\varepsilon}}_{\mathrm{eq}}^{(r)}=\left(\frac{2}{3} I::\langle\dot{\varepsilon} \otimes \dot{\varepsilon}\rangle^{(r)}\right)^{1 / 2},
\end{aligned}
$$

where $I$ is the fourth-order identity tensor. The standard deviations of the equivalent magnitudes over the whole polycrystal are defined as:

$$
\begin{aligned}
& \operatorname{SD}\left(\sigma_{\text {eq }}\right)=\sqrt{\overline{\bar{\Sigma}}_{\text {eq }}^{2}-\Sigma_{\text {eq }}^{2}}, \\
& \operatorname{SD}\left(\dot{\varepsilon}_{\text {eq }}\right)=\sqrt{\overline{\dot{E}}_{\text {eq }}^{2}-\dot{E}_{\text {eq }}^{2}},
\end{aligned}
$$

where

$$
\begin{aligned}
& \overline{\bar{\Sigma}}_{\mathrm{eq}}^{2}=\left\langle\left(\overline{\bar{\sigma}}_{\mathrm{eq}}^{(r)}\right)^{2}\right\rangle=\sum_{r} c^{(r)}\left(\overline{\bar{\sigma}}_{\mathrm{eq}}^{(r)}\right)^{2}, \\
& \overline{\overline{\dot{E}}}_{\mathrm{eq}}^{2}=\left\langle\left(\overline{\overline{\dot{\varepsilon}}}_{\mathrm{eq}}^{(r)}\right)^{2}\right\rangle=\sum_{r} c^{(r)}\left(\overline{\overline{\dot{\varepsilon}}}_{\mathrm{eq}}^{(r)}\right)^{2} .
\end{aligned}
$$

Once the average second-order moments of the stress field over each SR grain $(r)$ are obtained, the implementation of the second-order procedure follows the work of Liu and Ponte Castañeda (2004). The covariance tensor of stress fluctuations in the SR grains of the linear comparison polycrystal is given by:

$$
C_{\sigma^{\prime}}^{(r)}=\left\langle\sigma^{\prime} \otimes \sigma^{\prime}\right\rangle^{(r)}-\sigma^{\prime(r)} \otimes \sigma^{\prime(r)}
$$

The average and the average fluctuation of resolved shear stress on slip system $(k)$ of SR grain $(r)$ are given by:

$$
\begin{aligned}
& \bar{\tau}^{k(r)}=m^{k(r)}: \sigma^{\prime(r)}, \\
& \hat{\tau}^{k(r)}=\bar{\tau}^{k(r)} \pm\left(m^{k(r)}: C_{\sigma^{\prime}}^{(r)}: m^{k(r)}\right)^{1 / 2},
\end{aligned}
$$


where the positive (negative) branch should be selected if $\bar{\tau}^{k(r)}$ is positive (negative). The slip potential associated with slip system $(k)$ of the nonlinear polycrystal is defined as:

$$
\phi^{k}(\tau)=\frac{\tau_{\mathrm{o}}^{k}}{n+1}\left(\frac{|\tau|}{\tau_{\mathrm{o}}^{k}}\right)^{n+1}
$$

Two scalar magnitudes associated with each slip system $(k)$ of each SR grain $(r)$ are defined by:

$$
\begin{aligned}
\alpha^{k(r)} & =\frac{\phi^{\prime k(r)}\left(\hat{\tau}^{k(r)}\right)-\phi^{\prime k(r)}\left(\bar{\tau}^{k(r)}\right)}{\hat{\tau}^{k(r)}-\bar{\tau}^{k(r)}}, \\
e^{k(r)} & =\phi^{\prime k(r)}\left(\bar{\tau}^{k(r)}\right)-\alpha^{k(r)} \bar{\tau}^{k(r)},
\end{aligned}
$$

where $\phi^{\prime k}(\tau)=\mathrm{d} \phi^{k} / \mathrm{d} \tau(\tau)$. The linearized local behavior associated with SR grain $(r)$ is then given by:

$$
\dot{\varepsilon}^{(r)}=M_{\mathrm{SO}}^{(r)}: \sigma^{\prime(r)}+\dot{\varepsilon}_{\mathrm{SO}}^{o(r)}
$$

with

$$
\begin{aligned}
& M_{\mathrm{SO}}^{(r)}=\sum_{k} \alpha^{k(r)}\left(m^{k(r)} \otimes m^{k(r)}\right), \\
& \dot{\varepsilon}_{\mathrm{SO}}^{o(r)}=\sum_{k} e^{k(r)} m^{k(r)} .
\end{aligned}
$$

Once the linear comparison polycrystal is defined by (66-67), different secondorder estimates of the effective behavior of the nonlinear aggregate can be obtained. Approximating the potential of the nonlinear polycrystal in terms of the potential of the linear comparison polycrystal and a suitable measure of the error, Liu and Ponte Castañeda (2004) generated the following expression (corresponding to the so-called energy version of the second-order theory) for the effective potential of the nonlinear polycrystal:

$$
\bar{U}\left(\Sigma^{\prime}\right)=\sum_{r} c^{(r)} \sum_{k}\left\{\phi^{k(r)}\left(\hat{\tau}^{k(r)}\right)+\phi^{k(r)}\left(\bar{\tau}^{k(r)}\right)\left(\bar{\tau}^{k(r)}-\hat{\tau}^{k(r)}\right)\right\}
$$

from where the effective response of the homogenized polycrystal can be obtained as $\dot{E}=\partial \bar{U}\left(\Sigma^{\prime}\right) / \partial \Sigma^{\prime}$. The alternate constitutive equation version of the second-order theory simply consists in making use of the effective stress-strainrate relations for the linear comparison polycrystal, in which case, e.g., the effective strain is obtained as:

$$
\dot{E}=\sum_{r} c^{(r)} \sum_{k} m^{k(r)} \phi^{\prime k(r)}\left(\bar{\tau}^{k(r)}\right)
$$


Both versions of the SO theory give slightly different results, depending on nonlinearity and local anisotropic contrast. Such gap is relatively small compared with the larger differences obtained with the different SC approaches. The "constitutive equation" version is in principle less rigorous since it does not derive from a potential function, but has the advantage that can be obtained by simply following the affine algorithm described in the previous sections, using the linearized moduli defined in (66-67). Therefore, it is the adequate choice to be implemented in the VPSC code.

\subsubsection{Numerical Implementation}

To illustrate the use of the self-consistent formulation, we describe here the steps required to predict the local and overall viscoplastic response of a polycrystal. Starting for convenience with an initial Taylor guess, i.e., $\dot{\varepsilon}^{(r)}=\dot{E}$ for all grains, we solve the following nonlinear equation to get $\sigma^{\prime(r)}$ :

$$
\left.\dot{E}=\dot{\gamma}_{\mathrm{o}} \sum_{k} m^{k(r)} \frac{m^{k(r)}: \sigma^{\prime(r)}}{\tau_{\mathrm{o}}^{k(r)}}\right)^{n} \times \operatorname{sgn}\left(m^{k(r)}: \sigma^{\prime(r)}\right),
$$

and we use an appropriate first-order linearization scheme to obtain initial values of $M^{(r)}$ and $\dot{\varepsilon}^{o(r)}$, for each SR grain $(r)$. Next, initial guesses for the macroscopic moduli $\bar{M}$ and $\dot{E}^{o}$ are obtained (usually as simple averages of the local moduli). With them and the applied strain-rate, the initial guess for the macroscopic stress $\Sigma^{\prime}$ can be obtained (5), while the Eshelby tensors $S$ and $\Pi$ can be calculated using the macroscopic moduli and the ellipsoidal shape of the SR grains, by means of the procedure described in Sect. 2.1.1. Subsequently, the interaction tensor $\tilde{M}$ (30), and the localization tensors $B^{(r)}$ and $b^{(r)}$ (32 and 33), can be calculated as well. With these tensors, new estimates of $\bar{M}$ and $\dot{E}^{o}$ are obtained by solving iteratively the self-consistent equations (36) (for a unique grain shape) or (37) (for a distribution of grain shapes). After achieving convergence on the macroscopic moduli (and, consequently, also on the macroscopic stress and the interaction and localization tensors), a new estimation of the average grain stresses can be obtained, using the localization relation (31). If the recalculated average grain stresses are different (within certain tolerance) from the input values, a new iteration should be started, until convergence is reached. If the chosen linearization scheme is the second-order formulation, an additional loop on the linearized moduli is needed, using the improved estimates of the second-order moments of the stress in the grains, obtained by means of the methodology described in Sect. 2.1.5 and the Appendix. This additional loop roughly increases the calculation time by one order of magnitude with respect to first-order linearizations. When the iterative procedure is completed, the average shear-rates on the slip system $(k)$ in each grain $(r)$ are calculated as:

$$
\left.\dot{\gamma}^{k(r)}=\dot{\gamma}_{\mathrm{o}} \frac{m^{k(r)}: \sigma^{\prime(r)}}{\tau_{\mathrm{o}}^{k(r)}}\right)^{n} \times \operatorname{sgn}\left(m^{k(r)}: \sigma^{\prime(r)}\right) .
$$


These average shear-rates are in turn used to calculate the lattice rotation-rates associated with each SR grain:

$$
\dot{\omega}_{i j}^{(r)}=\dot{\Omega}_{i j}+\tilde{\dot{\omega}}_{i j}^{(r)}-\dot{\omega}_{i j}^{p(r)},
$$

with [c.f. (2)]:

$$
\dot{\omega}_{i j}^{p(r)}=\sum_{k} \alpha_{i j}^{k(r)} \dot{\gamma}^{k(r)}
$$

where $\alpha_{i j}^{k(r)}$ is the uniform antisymmetric Schmid tensor of system $(k)$ in SR grain $(r)$.

It is worth noting that in the case of first-order approximations, although the second-order moments are not needed to readjust iteratively the linearized behavior of the SR grains, the average field fluctuations associated with the converged values of the effective moduli can be obtained as well, after convergence is reached.

The above numerical scheme can be used to predict texture development, by applying viscoplastic deformation to the polycrystal in incremental steps. The latter is done by assuming constant rates during a time interval $\Delta t$ (such that $\dot{E} \Delta t$ corresponds to a macroscopic strain increment of the order of a few percents) and using: (1) the strain-rates and rotation-rates (times $\Delta t$ ) to update the shape and orientation of the SR grains, and (2) the shear-rates (times $\Delta t$ ) to update the critical stress of the deformation systems due to strain hardening, after each deformation increment. Using extended Voce law (Tomé et al. 1984), the evolution of the threshold stress with accumulated shear strain in each grain is given by:

$$
\tau^{* k(r)}=\tau_{\mathrm{oo}}^{k}+\left(\tau_{1}^{k}+\theta_{1}^{k} \Gamma^{(r)}\right)\left(1-\exp \left(-\Gamma^{(r)}\left|\theta_{\mathrm{o}}^{k} / \tau_{1}^{k}\right|\right)\right),
$$

where $\Gamma^{(r)}$ is the total accumulated shear in the grain; $\tau_{\mathrm{oo}}^{k}, \tau_{1}^{k}, \theta_{\mathrm{o}}^{k}$, and $\theta_{1}^{k}$ are the initial threshold stress, initial hardening rate, asymptotic hardening rate, and backextrapolated threshold stress, respectively. In addition, we allow for the possibility of "self" and "latent" hardening by defining coupling coefficients $h^{k k^{\prime}}$, which empirically account for the obstacles that new dislocations (or twins) associated with system $k^{\prime}$ represent for the propagation of dislocations (or twins) on system $k$. The increase in the threshold stress is calculated as:

$$
\Delta \tau_{\mathrm{o}}^{k(r)}=\frac{\mathrm{d} \tau^{* k(r)}}{\mathrm{d} \Gamma^{(r)}} \sum_{k^{\prime}} h^{k k^{\prime}} \dot{\gamma}^{k^{\prime}(r)} \Delta t .
$$

Note that the above explicit update schemes rely on the fact that the orientation and hardening variables evolve slowly within the adopted time interval. Otherwise, $\Delta t$ should be chosen smaller. 


\subsection{FFT-Based Formalism}

The FFT-based full-field formulation for viscoplastic polycrystals is conceived for periodic unit cells, provides an "exact" solution (within the limitations imposed by the unavoidable discretization of the problem and the iterative character of the numerical algorithm, see below) of the governing equations, and has better numerical performance than a finite element calculation for the same purpose and resolution (at least when comparing sequential implementations of both methods). It was originally developed (Moulinec and Suquet 1994, 1998; Michel et al. 2000) as a fast algorithm to compute the elastic and elastoplastic effective and local response of composites, and later adapted (Lebensohn 2001; Lebensohn et al. 2004b, 2008) to deal with the viscoplastic deformation of three-dimensional (3D) power-law polycrystals. It shares some common characteristics with the phase field method, although it is limited to what in phase field jargon is known as long-range interactions (Chen 2004), since no heterogeneous chemical energy term is involved in the mechanical response and/or microstructure evolution of a single-phase polycrystal. Recently, a similar kind of phase field analysis was proposed (Wang et al. 2002) to obtain the local fields in elastically heterogeneous polycrystals. The FFT-based approach, however, is not restricted to linear behaviors. Problems involving nonlinear materials (e.g., viscoplastic polycrystals) are treated similarly to a linear problem, using the concept of linear reference material.

Briefly, the viscoplastic FFT-based formulation consists in iteratively adjusting a compatible strain-rate field, related to an equilibrated stress field through a constitutive potential, such that the average of local work-rates is minimized. The method is based on the fact that the local mechanical response of a heterogeneous medium can be calculated as a convolution integral between Green functions associated with appropriate fields of a linear reference homogeneous medium and the actual heterogeneity field. For periodic media, use can be made of the Fourier transform to reduce convolution integrals in real space to simple products in Fourier space. Thus, the FFT algorithm can be used to transform the heterogeneity field into Fourier space and, in turn, to get the mechanical fields by transforming that product back to real space. However, the actual heterogeneity field depends precisely on the a priori unknown mechanical fields. Therefore, an iterative scheme has to be implemented to obtain, upon convergence, a compatible strain-rate field and a stress field in equilibrium.

\subsubsection{Periodic Unit Cell: Green Function Method}

A periodic unit cell representing the polycrystal is discretized into $N_{1} \times N_{2} \times N_{3}$ Fourier points. This discretization determines a regular grid in the Cartesian space $\left\{\mathbf{x}^{\mathbf{d}}\right\}$ and a corresponding grid in Fourier space $\left\{\xi^{\mathbf{d}}\right\}$. Velocities and tractions along the boundary of the unit cell are left undetermined. A velocity-gradient $V_{i, j}$ (which can be decomposed into a symmetric strain-rate and a antisymmetric rotation-rate: $\left.V_{i, j}=\dot{E}_{i j}+\dot{\Omega}_{i j}\right)$ is imposed to the unit cell. The local strain-rate field is a function 
of the local velocity field, i.e., $\dot{\varepsilon}_{i j}\left(v_{k}(\mathbf{x})\right)$, and can be split into its average and a fluctuation term: $\dot{\varepsilon}_{i j}\left(v_{k}(\mathbf{x})\right)=\dot{E}_{i j}+\tilde{\dot{\varepsilon}}_{i j}\left(\tilde{v}_{k}(\mathbf{x})\right)$, where $v_{i}(\mathbf{x})=\dot{E}_{i j} x_{j}+\tilde{v}_{i}(\mathbf{x})$. By imposing periodic boundary conditions, the velocity fluctuation field $\tilde{v}_{k}(\mathbf{x})$ is assumed to be periodic across the boundary of the unit cell, while the traction field is antiperiodic, to meet equilibrium on the boundary between contiguous unit cells.

The local constitutive relation between the strain-rate $\dot{\varepsilon}_{i j}(\mathbf{x})$ and the deviatoric stress $\sigma_{i j}^{\prime}(\mathbf{x})$ is given by the same rate-sensitivity relation used within the VPSC framework (1). Let us choose a fourth-order tensor $L^{o}$ to be the stiffness of a linear reference medium (the choice of $L^{o}$ can be quite arbitrary, but the speed of convergence of the method will depend on this choice) and define the polarization field $\varphi_{i j}(\mathbf{x})[$ c.f. (10)] as:

$$
\varphi_{i j}(\mathbf{x})=\tilde{\sigma}_{i j}^{\prime}(\mathbf{x})-L_{i j k l}^{o} \tilde{\dot{\varepsilon}}_{k l}(\mathbf{x}) .
$$

Then, the Cauchy stress deviation can be written as:

$$
\tilde{\sigma}_{i j}(\mathbf{x})=L_{i j k l}^{o} \tilde{\dot{\varepsilon}}_{k l}(\mathbf{x})+\varphi_{i j}(\mathbf{x})+\tilde{\sigma}^{m}(\mathbf{x}) \delta_{i j} .
$$

Combining (77) with the equilibrium $\left(\sigma_{i j, j}(\mathbf{x})=\tilde{\sigma}_{i j, j}(\mathbf{x})=0\right)$, the incompressibility condition, and the relation $\tilde{\dot{\varepsilon}}_{i j}(\mathbf{x})=\frac{1}{2}\left(\tilde{v}_{i, j}(\mathbf{x})+\tilde{v}_{j, i}(\mathbf{x})\right)$ :

$$
\mid \begin{aligned}
& L_{i j k l}^{o} \tilde{v}_{k, l j}(\mathbf{x})+\tilde{\sigma}_{, i}^{m}(\mathbf{x})+\varphi_{i j, j}(\mathbf{x})=0, \\
& \tilde{v}_{k, k}(\mathbf{x})=0 .
\end{aligned}
$$

This system of differential equations is formally equivalent to system (9). However, both systems actually differ in that: (1) the HEM's stiffness modulus $\bar{L}$ of (9) is replaced in (78) by the stiffness of a linear reference medium $L^{o}$, and (2) the polarization field in (78) has in general nonvanishing values throughout the unit cell and is periodic (owing to the unit cell's periodicity), while the polarization field in (9) vanishes outside the domain of the inclusion. The auxiliary system involving Green functions is then given by [c.f. (13)]:

$$
\mid \begin{aligned}
& L_{i j k l}^{o} G_{k m, l j}\left(\mathbf{x}-\mathbf{x}^{\prime}\right)+H_{m, i}\left(\mathbf{x}-\mathbf{x}^{\prime}\right)+\delta_{i m} \delta\left(\mathbf{x}-\mathbf{x}^{\prime}\right)=0, \\
& G_{k m, k}\left(\mathbf{x}-\mathbf{x}^{\prime}\right)=0
\end{aligned}
$$

After some manipulation, the convolution integrals that give the velocity and velocity-gradient deviation fields are:

$$
\begin{gathered}
\tilde{v}_{k}(x)=\int_{R^{3}} G_{k i, j}\left(x-x^{\prime}\right) \varphi_{i j}\left(x^{\prime}\right) \mathrm{d} x^{\prime}, \\
\tilde{v}_{i, j}(x)=\int_{R^{3}} G_{i k, j l}\left(x-x^{\prime}\right) \varphi_{k l}\left(x^{\prime}\right) \mathrm{d} x^{\prime} .
\end{gathered}
$$


Convolution integrals in direct space are simply products in Fourier space:

$$
\begin{aligned}
\hat{\tilde{v}}_{k}(\xi) & =(-i \xi j) \hat{G}_{k i}(\xi) \hat{\varphi}_{i j}(\xi), \\
\hat{\tilde{\tilde{v}}}_{i, j}(\xi) & =\hat{\Gamma}_{i j k l}(\xi) \hat{\varphi}_{k l}(\xi),
\end{aligned}
$$

where the symbol " "»" indicates a Fourier transform. The Green operator in (83) is defined as $\Gamma_{i j k l}=G_{i k, j l}$. The tensors $\hat{G}_{i j}(\xi)$ and $\hat{\Gamma}_{i j k l}(\xi)$ can be calculated by taking Fourier transform to system (79):

$$
\mid \begin{aligned}
& \xi_{l} \xi_{j} L_{i j k l}^{o} \hat{G}_{k m}(\xi)+i \xi_{i} \hat{H}_{m}(\xi)=\delta_{i m} \\
& \xi_{k} \hat{G}_{k m}(\xi)=0
\end{aligned} .
$$

Defining the $3 \times 3$ matrix $\mathbf{A}_{i k}^{\prime}(\boldsymbol{\xi})=\xi_{l} \xi_{j} L_{i j k l}^{o}$, and the $4 \times 4$ matrix $\mathbf{A}(\boldsymbol{\xi})$ :

$$
\mathbf{A}(\boldsymbol{\xi})=\left|\begin{array}{cccc}
A_{11}^{\prime} & A_{12}^{\prime} & A_{13}^{\prime} & \xi_{1} \\
A_{21}^{\prime} & A_{22}^{\prime} & A_{23}^{\prime} & \xi_{2} \\
A_{31}^{\prime} & A_{32}^{\prime} & A_{33}^{\prime} & \xi_{3} \\
\xi_{1} & \xi_{2} & \xi_{3} & 0
\end{array}\right|,
$$

we obtain from (84) [c.f. (14 and 15)]:

$$
\hat{G}_{i j}(\xi)=\mathbf{A}_{i j}^{-1}(i, j=1,3)
$$

and

$$
\hat{\Gamma}_{i j k l}(\xi)=-\xi_{j} \xi_{l} \hat{G}_{i k}(\xi)
$$

\subsubsection{FFT-Based Algorithm}

Assigning initial guess values to the strain-rate field in the regular grid $\left\{\mathbf{x}^{\mathbf{d}}\right\}$ (e.g., $\left.\tilde{\varepsilon}_{i j}^{o}\left(\mathbf{x}^{\mathbf{d}}\right)=0 \Rightarrow \dot{\varepsilon}_{i j}^{o}\left(\mathbf{x}^{\mathbf{d}}\right)=\dot{E}_{i j}\right)$, and computing the corresponding stress field $\sigma_{i j}^{\prime o}\left(\mathbf{x}^{\mathbf{d}}\right)$ from the local constitutive relation (1) (which requires to know the initial values of the critical stresses $\tau_{\mathrm{o}}^{s}\left(\mathbf{x}^{\mathbf{d}}\right)$, and the Schmid tensors $m_{i j}^{s}\left(\mathbf{x}^{\mathbf{d}}\right)$, e.g., from an orientation image, in which the image's pixels coincide with the Fourier grid), allow us to obtain an initial guess for the polarization field in direct space $\varphi_{i j}^{o}\left(\mathbf{x}^{\mathbf{d}}\right)$ (76), which in turn can be Fourier-transformed to obtain $\hat{\varphi}_{i j}^{o}\left(\xi^{\mathbf{d}}\right)$. Furthermore, assuming that $\lambda_{i j}^{o}\left(\mathbf{x}^{\mathbf{d}}\right)=\sigma_{i j}^{o}\left(\mathbf{x}^{\mathbf{d}}\right)$ is the initial guess for a field of Lagrange multipliers associated with the compatibility constraints, the iterative procedure based on Augmented Lagrangians proposed by Michel et al. (2000) reads as follows. With the polarization field after iteration $\mathrm{n}$ being known, the $n+1$-th iteration starts by computing the new guess for the kinematically admissible strain-rate deviation field:

$$
\hat{\tilde{d}}_{i j}^{n+1}\left(\xi^{\mathbf{d}}\right)=-\hat{\Gamma}_{i j k l}^{\mathrm{sym}}\left(\xi^{\mathbf{d}}\right) \hat{\varphi}_{k l}^{n}\left(\xi^{\mathbf{d}}\right), \quad \forall \xi^{\mathbf{d}} \neq 0 ; \quad \text { and } \quad \hat{\tilde{d}}_{i j}^{n+1}(\mathbf{0})=0,
$$


where $\hat{\Gamma}_{i j k l}^{\text {sym }}$ is the Green operator, appropriately symmetrized. The corresponding field in real space is thus obtained by application of the inverse FFT, i.e.,

$$
\tilde{d}_{i j}^{n+1}\left(\mathbf{x}^{\mathbf{d}}\right)=f f t^{-1}\left\{\hat{\tilde{d}}_{i j}^{n+1}\left(\xi^{\mathbf{d}}\right)\right\},
$$

and the new guess for the deviatoric stress field is calculated from (omitting subindices):

$$
\begin{aligned}
& \left.\sigma^{\prime n+1}\left(\mathbf{x}^{\mathbf{d}}\right)+L^{o}: \dot{\gamma}_{\mathrm{o}} \sum_{k} m^{k}\left(\mathbf{x}^{\mathbf{d}}\right) \frac{m^{k}\left(\mathbf{x}^{\mathbf{d}}\right): \sigma^{\prime n+1}\left(\mathbf{x}^{\mathbf{d}}\right)}{\tau^{k}\left(\mathbf{x}^{\mathbf{d}}\right)}\right)^{n} \times \operatorname{sgn}\left(m^{k}\left(\mathbf{x}^{\mathbf{d}}\right): \sigma^{\prime n+1}\left(\mathbf{x}^{\mathbf{d}}\right)\right) \\
& =\lambda^{n}\left(\mathbf{x}^{\mathbf{d}}\right)+L^{o}:\left(\dot{E}+\tilde{d}^{n+1}\left(\mathbf{x}^{\mathbf{d}}\right)\right) .
\end{aligned}
$$

The iteration is completed with the calculation of the new guess of the Lagrange multiplier field:

$$
\lambda^{n+1}\left(\mathbf{x}^{\mathbf{d}}\right)=\lambda^{n}\left(\mathbf{x}^{\mathbf{d}}\right)+L^{o}:\left(\tilde{\dot{\varepsilon}}^{n+1}\left(\mathbf{x}^{\mathbf{d}}\right)-\tilde{d}^{n+1}\left(\mathbf{x}^{\mathbf{d}}\right)\right)
$$

Equations (90 and 91) guarantee the convergence of: $\dot{\varepsilon}\left(\mathbf{x}^{\mathbf{d}}\right)$ (i.e., the strain-rate field related with the stress through the constitutive equation) toward $d\left(\mathbf{x}^{\mathbf{d}}\right)$ (i.e., the kinematically admissible strain-rate field) to fulfill compatibility, and the Lagrange multiplier field $\lambda\left(\mathbf{x}^{\mathbf{d}}\right)$ toward the stress field $\sigma^{\prime}\left(\mathbf{x}^{\mathbf{d}}\right)$ to fulfill equilibrium.

Upon convergence, the microstructure can be updated using an explicit scheme, as follows. The resulting strain-rate field, and the shear-rate field, i.e.

$$
\left.\dot{\gamma}^{k}\left(\mathbf{x}^{\mathbf{d}}\right)=\dot{\gamma}_{\mathrm{o}} \frac{m^{k}\left(\mathbf{x}^{\mathbf{d}}\right): \sigma^{\prime}\left(\mathbf{x}^{\mathbf{d}}\right)}{\tau^{k}\left(\mathbf{x}^{\mathbf{d}}\right)}\right)^{n} \times \operatorname{sgn}\left(m^{k}\left(\mathbf{x}^{\mathbf{d}}\right): \sigma^{\prime}\left(\mathbf{x}^{\mathbf{d}}\right)\right)
$$

can be assumed to be constant during a time interval $[t, t+\Delta t]$. The macroscopic and local strain increments are then calculated as: $\Delta E_{i j}=\dot{E}_{i j} \times \Delta t$ and $\Delta \varepsilon_{i j}\left(\mathbf{x}^{\mathbf{d}}\right)=$ $\dot{\varepsilon}_{i j}\left(\mathbf{x}^{\mathbf{d}}\right) \times \Delta t$, and the local crystallographic orientations are updated according to the following local lattice rotation:

$$
\omega_{i j}\left(\mathbf{x}^{\mathbf{d}}\right)=\left(\dot{\Omega}_{i j}\left(\mathbf{x}^{\mathbf{d}}\right)+\tilde{\dot{\omega}}_{i j}\left(\mathbf{x}^{\mathbf{d}}\right)-\dot{\omega}_{i j}^{p}\left(\mathbf{x}^{\mathbf{d}}\right)\right) \times \Delta t,
$$

where $\dot{\omega}_{i j}^{p}\left(\mathbf{x}^{\mathbf{d}}\right)$ can be obtained from (2) and (92), and $\tilde{\dot{\omega}}\left(\mathbf{x}^{\mathbf{d}}\right)$ is obtained backtransforming the converged antisymmetric field:

$$
\hat{\tilde{\omega}}_{i j}\left(\xi^{\mathbf{d}}\right)=-\hat{\Gamma}_{i j k l}^{\text {antisym }}\left(\xi^{\mathbf{d}}\right) \hat{\varphi}_{k l}\left(\xi^{\mathbf{d}}\right), \quad \forall \xi^{\mathbf{d}} \neq 0 ; \quad \text { and } \quad \hat{\tilde{\omega}}_{i j}(\mathbf{0})=0
$$

The critical resolved shear stresses of the deformation systems associated with each material point should also be updated after each deformation increment due to strain 
hardening, e.g., in an analogous way as explained in Sect. 2.1.6 for the VPSC case (in terms a phenomenological Voce law) or with more sophisticated hardening laws based directly on dislocation densities. Note that, in the latter case, the possibility of calculating the intragranular misorientations would allow us to track the evolution of geometrically necessary dislocations (GND) densities explicitly, and, at the same time, introduce a length scale in the formulation (see, e.g., Acharya et al. 2003). This more elaborated treatment of hardening, however, is not going to be discussed further in this work.

After each time increment, the new position of the Fourier points can be determined calculating the velocity fluctuation term $\tilde{v}_{k}\left(\mathbf{x}^{\mathbf{d}}\right)$ back-transforming (82), and:

$$
X_{i}\left(\mathbf{x}^{\mathbf{d}}\right)=x_{i}^{d}+\left(\dot{E}_{i j} x_{j}^{d}+\tilde{v}_{i}\left(\mathbf{x}^{\mathbf{d}}\right)\right) \times \Delta t .
$$

Evidently, due to the heterogeneity of the medium, the set of convected Fourier points no longer forms a regular grid, after the very first deformation increment. A rigorous way of dealing with this situation was proposed by Lahellec et al. (2001) based on the particle-in-cell (PIC) method (Sulsky et al. 1995). In the example presented in Sects. 3 and 4 below, however, the following simplification was adopted. Neglecting the velocity fluctuation term in (95), the updated coordinates of the Fourier points can be approximated by:

$$
X_{i}\left(\mathbf{x}^{\mathbf{d}}\right) \stackrel{\sim}{=} x_{i}^{d}+\dot{E}_{i j} x_{j}^{d} \times \Delta t .
$$

In this way, the Fourier grid remains regular after each deformation increment. The distances between adjacent Fourier points, however, do change, following the variations of the unit cell dimensions, thus determining an "average stretching" of the grains, following the macroscopic deformation.

\section{Results}

\subsection{Validation of the Full-Field Formulation Using an Analytical Result}

Let us consider a model polycrystal consisting of columnar orthorhombic grains with symmetry axes aligned with the $x_{3}$ axis, such that, when loaded in antiplane mode with shearing direction along $x_{3}$, the only two slip systems that can be activated in the grains are those defined by the following Schmid tensors:

$$
m^{\mathrm{s}}=\frac{\left(\mathbf{e}_{1} \otimes \mathbf{e}_{3}+\mathbf{e}_{3} \otimes \mathbf{e}_{1}\right)}{2}, \quad m^{\mathrm{h}}=\frac{\left(\mathbf{e}_{2} \otimes \mathbf{e}_{3}+\mathbf{e}_{3} \otimes \mathbf{e}_{2}\right)}{2},
$$

where $\left\{\mathbf{e}_{1}, \mathbf{e}_{2}, \mathbf{e}_{3}\right\}$ is an orthonormal basis of crystallographic axes, and "s" and " $h$ " stand for soft and hard slip systems, respectively. If we further consider that 
$\mathbf{e}_{3}$ lies parallel to $x_{3}$, and the material is incompressible, the problem becomes two-dimensional (2D). The local stress and strain-rate are characterized by the 2D vectors with components $\sigma_{13}$ and $\sigma_{23}$, and $\dot{\varepsilon}_{13}$ and $\dot{\varepsilon}_{23}$ (denoted hereafter $\sigma_{1}$ and $\sigma_{2}$, and $\dot{\varepsilon}_{1}$ and $\dot{\varepsilon}_{2}$, respectively), and the viscous stiffness tensor $L=2 \mu$, by a $2 \mathrm{D}$ symmetric second-order tensor with diagonal components $2 \mu_{1313}$ and $2 \mu_{2323}$, and off-diagonal components $2 \mu_{1323}$ (denoted $2 \mu_{11}, 2 \mu_{22}$, and $2 \mu_{12}$, respectively). In addition, let us assume that the constituent grains exhibit a linear response:

$$
\dot{\varepsilon}(\mathbf{x})=L^{-1}: \sigma(\mathbf{x})=\left(\frac{1}{\tau_{\mathrm{o}}^{\mathrm{s}}} m^{\mathrm{s}} \otimes m^{\mathrm{s}}+\frac{1}{\tau_{\mathrm{o}}^{\mathrm{h}}} m^{\mathrm{h}} \otimes m^{\mathrm{h}}\right): \sigma(\mathbf{x}),
$$

with $\tau_{\mathrm{o}}^{\mathrm{s}}$ and $\tau_{\mathrm{o}}^{\mathrm{h}}$ being the viscosities of the soft and hard slip systems $\left(\tau_{\mathrm{o}}^{\mathrm{s}}<\tau_{\mathrm{o}}^{\mathrm{h}}\right)$. It can be shown that the behavior of such polycrystal is characterized by an effective $2 \mathrm{D}$ viscous stiffness tensor $\bar{L}=2 \bar{\mu}$ such that $\dot{E}=\bar{L}^{-1}: \Sigma$ (where $\Sigma$ and $\dot{E}$ are the 2D effective stress and strain-rate, respectively), such that (Dykhne 1970; Lurie and Cherkaev 1984):

$$
\operatorname{det}(\bar{\mu})=\bar{\mu}_{11} \times \bar{\mu}_{22}-\bar{\mu}_{12}^{2}=\tau_{\mathrm{o}}^{\mathrm{s}} \times \tau_{\mathrm{o}}^{\mathrm{h}} .
$$

In the particular case of an isotropic 2 D polycrystal, $\bar{\mu}_{11}=\bar{\mu}_{22}(=\bar{\mu})$ and $\bar{\mu}_{12}=0$, so that the effective shear modulus becomes:

$$
\bar{\mu}=\sqrt{\tau_{\mathrm{o}}^{\mathrm{s}} \times \tau_{\mathrm{o}}^{\mathrm{h}}}
$$

Note that the above result is independent of the $2 \mathrm{D}$ microstructure as far as it remains isotropic. This analytical result can be used for validating the FFT-based formulation. Let us consider the periodic 2D two-phase composite shown in Fig. 1a (Lebensohn et al. 2005), whose unit cell consists of four square grains, with the crystallographic orientations of the two pairs of opposite grains (i.e., each pair shearing only the central vertex) being characterized by angles $+45^{\circ}$ and $-45^{\circ}$, respectively (note that the orientation of each $2 \mathrm{D}$ crystal is fully characterized by the angle between the crystal direction $\mathbf{e}_{1}$ and the sample direction $x_{1}$ ). The antiplane deformation of this unit cell for an applied strain-rate of the form $\dot{E}=\left(\dot{E}_{13}, 0\right)$ was solved numerically using different discretizations: 64, 128, 256, and 512 Fourier points along each direction (i.e., 1,024, 4,096, 16,384, 65,536 Fourier points per grain), for a contrast of $\tau_{\mathrm{o}}^{\mathrm{h}} / \tau_{\mathrm{o}}^{\mathrm{s}}=25$, which gives theoretical polycrystal viscosity of $\bar{\mu} / \tau_{\mathrm{o}}^{\mathrm{s}}=5$. Figure 2 shows the relative deviations of the polycrystal viscosities calculated with the FFT-based model from the theoretical value, as the number of iterations of the FFT-based method increases. It is seen that: (1) the convergence of $\bar{\mu}^{\mathrm{FFT}}$ toward its theoretical value is rather good, although it saturates at different levels, depending on the number of discretization points used; (2) the precision of the FFT solution can be increased by refining appropriately the Fourier grid. This is due to the fact that a more refined grid provides a higher spatial resolution to represent the strong gradients and jumps of the local fields, localized at grain boundaries (see discussion of Fig. 1c-f below). 
a

configuration

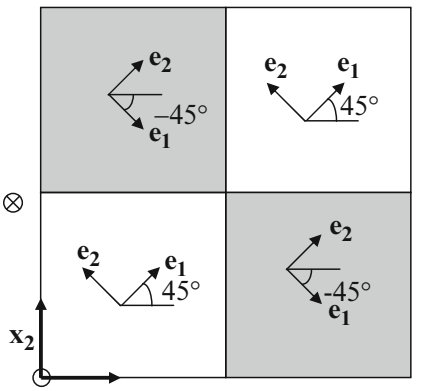

$\mathbf{x}_{3} \quad \mathbf{x}_{1}$

C $\quad \dot{\varepsilon}_{13}(\mathrm{X}) / \dot{\mathrm{E}}_{13}$

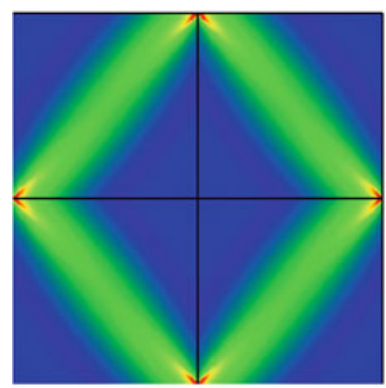

e

$$
\sigma_{13}(\mathrm{X}) / \Sigma_{13}
$$

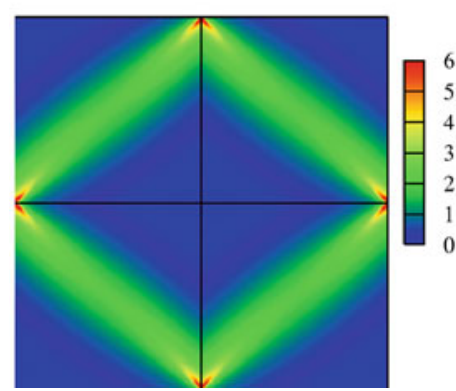

b soft system relative activity

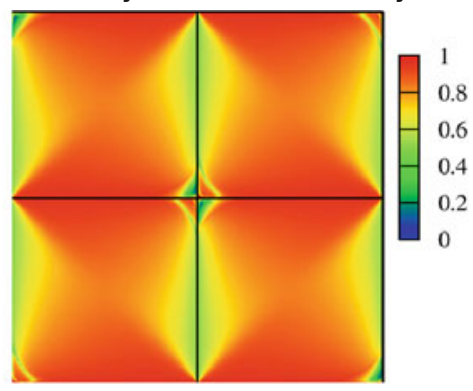

d $\quad \dot{\varepsilon}_{23}(\mathrm{X}) / \dot{\mathrm{E}}_{23}$

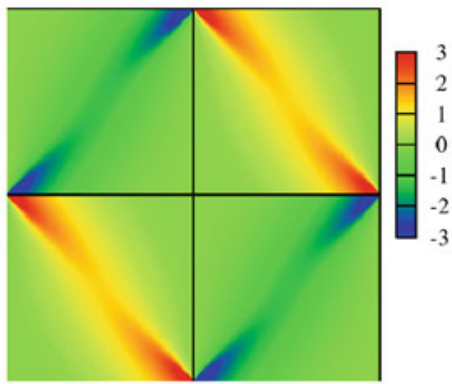

f $\quad \sigma_{23}(\mathrm{X}) / \Sigma_{13}$

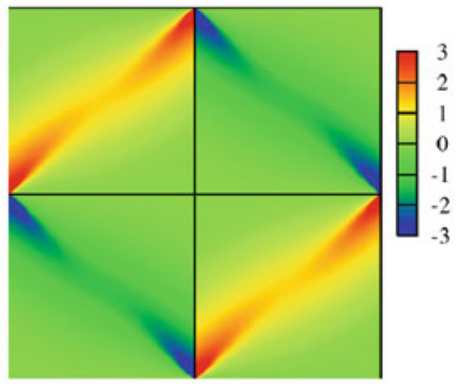

Fig. 1 (a) Two-dimensional two-phase isotropic unit cell undergoing antiplane deformation. FFTbased results of: (b) relative activity field of the soft slip system. Shear components 13 and 23 of the (c-d) strain-rate and (e-f) stress components, normalized with the value of the corresponding macroscopic component

On the other hand, since one of our goals is to understand the influence of the microstructure on the distribution of the stress and strain-rate fields, it is important to assess the precision of the FFT-based results also at the local scale. In this context, a great advantage of microstructures with only two phases is that the phase averages of 
Fig. 2 Relative deviations from the theoretical (th) value $\left(\bar{\mu} / \tau_{\mathrm{o}}^{\mathrm{s}}=5\right)$ of the polycrystal's viscosity predicted with the present formulation (FFT) for different grid refinements, in the case 2D antiplane deformation of the isotropic unit cell of Fig. 1a, consisting of grains having linear behavior (98) with $\tau_{\mathrm{o}}^{\mathrm{h}} / \tau_{\mathrm{o}}^{\mathrm{s}}=25$

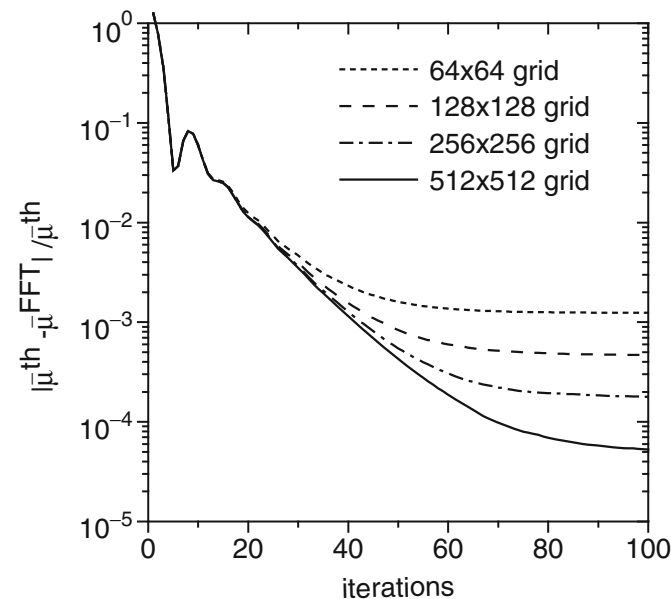

the localization tensors $A(\mathbf{x})$ and $B(\mathbf{x})$, defined by the expressions $\dot{\varepsilon}(\mathbf{x})=A(\mathbf{x})$ : $\dot{E}$ and $\sigma(\mathbf{x})=B(\mathbf{x}): \Sigma$ can be easily calculated analytically as (Lebensohn et al. 2005):

$$
\begin{aligned}
& \langle A\rangle_{1}=\frac{1}{c_{1}}\left(L_{1}-L_{2}\right)^{-1}:\left(\bar{L}-L_{2}\right), \\
& \langle B\rangle_{1}=\frac{1}{c_{1}}\left(M_{1}-M_{2}\right)^{-1}:\left(\bar{M}-M_{2}\right),
\end{aligned}
$$

where $c_{i}$ and $\langle\cdot\rangle_{i}$ denote volume fraction and average over phase $i=1,2$, respectively, and the local and effective compliance tensors are given by $M_{i}=L_{i}^{-1}$ and $\bar{M}=\bar{L}^{-1}$, respectively (similar relations can be obtained for $\langle A\rangle_{2}$ and $\langle B\rangle_{2}$ by interchanging indices 1 and 2. For isotropic microstructures with linear behavior as the one considered here, since $\bar{L}$ is microstructure-independent, the above expressions are also microstructure-independent. Using (100), the phase-average localization tensors for the considered microstructure are given by:

$$
\langle A\rangle_{1}=\langle B\rangle_{2}=\left(\begin{array}{cc}
1 & +\alpha \\
+\alpha & 1
\end{array}\right),\langle A\rangle_{2}=\langle B\rangle_{1}=\left(\begin{array}{cc}
1 & -\alpha \\
-\alpha & 1
\end{array}\right),
$$

where the indices 1 and 2 were used for phases at angles $+45^{\circ}$ and $-45^{\circ}$, respectively, and:

$$
\alpha=1-\frac{2}{\left(1+\sqrt{\tau_{\mathrm{o}}^{\mathrm{h}} / \tau_{\mathrm{o}}^{\mathrm{s}}}\right)} .
$$

The above analytical expressions can be used to evaluate the accuracy reached with the FFT-based simulations at phase-average level. Table 1 shows the values of $\alpha$ obtained for different grid refinements (Lebensohn et al. 2005). The agreement 
Table 1 Value of parameter $\alpha$ (103) predicted for different grid refinements and relative error with respect to the theoretical value $(\alpha=2 / 3)$

\begin{tabular}{lll}
\hline Grid & $\alpha$ & Relative error \\
\hline $64 \times 64$ & 0.666021 & $9.69 \times 10^{-4}$ \\
$128 \times 128$ & 0.666340 & $4.90 \times 10^{-4}$ \\
$256 \times 256$ & 0.666452 & $1.87 \times 10^{-4}$ \\
$512 \times 512$ & 0.666734 & $1.01 \times 10^{-4}$ \\
\hline
\end{tabular}

between the FFT-based predictions and the theoretical values is as good as for the corresponding effective viscosities shown in Fig. 2 and, like before, is better for more refined Fourier grids.

Finally, we show the predicted local strain-rate and stress fields. Figure 1c, d shows, respectively, the 13 and 23 components of the strain-rate field (normalized with the value of the applied macroscopic shear-rate), while Fig. 1e, f shows the analogous stress components (normalized with the resulting macroscopic shear stress). The first observation concerns the formation of localization bands (both of stress and strain-rate), which are normal to $\mathbf{e}_{1}$ (i.e., the normal to the shear plane of the soft slip system) in every grain. These bands go through quadruple points, where the 13 components ( 13 is the only nonvanishing component at polycrystal level) of the stress and strain-rate fields reach their maximum values. Meanwhile, the 23 components of the local fields also have non-negligible values along the localization bands, with alternating signs in the phases, such that the strain-rate is negative where the stress is positive and vice versa. This alternation is consistent with the plus and minus signs preceding $\alpha$ in (102) and also give vanishing average 23 stress and strain-rate components at macoscopic level. It is also worth noting that the corresponding stress and strain-rate field components are related by a $90^{\circ}$ rotation. Such symmetry is evidently related to the fact that a $2 \mathrm{D}$ divergence-free field has the property of transforming into a curl-free field when rotated by $90^{\circ}$ (Dykhne 1970). Note for instance that while stress equilibrium requires continuity of the 23 stress component through the "horizontal" grain boundaries (as in Fig. 1f), strain compatibility requires continuity of the 23 strain-rate component through the "vertical" grain boundaries. To complete the analysis, Fig. 1b shows the complicated pattern of the field of relative activity of the soft slip system, associated with the local and macroscopic response discussed above.

\subsection{Validation of Mean-Field Formulations Using Full-Field Computations}

The advantage of using field fluctuation information in nonlinear homogenization schemes to get improved predictions of the mechanical behavior and texture development of viscoplastic polycrystals becomes evident as the heterogeneity (contrast in local properties) increases. The two possible sources of heterogeneity in singlephase viscoplastic aggregates are the nonlinearity of the material's response and the 
local anisotropy of the constituent single crystals. To study the influence of both sources of heterogeneity, we show here examples of self-consistent calculations on different material systems: (1) fcc aggregates (compatible with, e.g., polycrystalline copper) with fixed local anisotropy (given by the - rather mild - range of variation of the Taylor factor of individual grains) and variable rate-sensitivity, and (2) hexagonal polycrystals with four and two soft independent slip systems, and orthorhombic aggregates (compatible with Ti deforming at high temperature, ice, and olivine, respectively), with mild nonlinear behavior and variable local anisotropy, given by the ratio between the threshold resolved shear stresses associated with hard and soft slip modes (Lebensohn et al. 2007).

The prediction of the effective properties of a random fcc polycrystal as the rate-sensitivity of the material decreases is a classical benchmark for the different nonlinear SC approaches. Figure 3 a shows a comparison between average Taylor
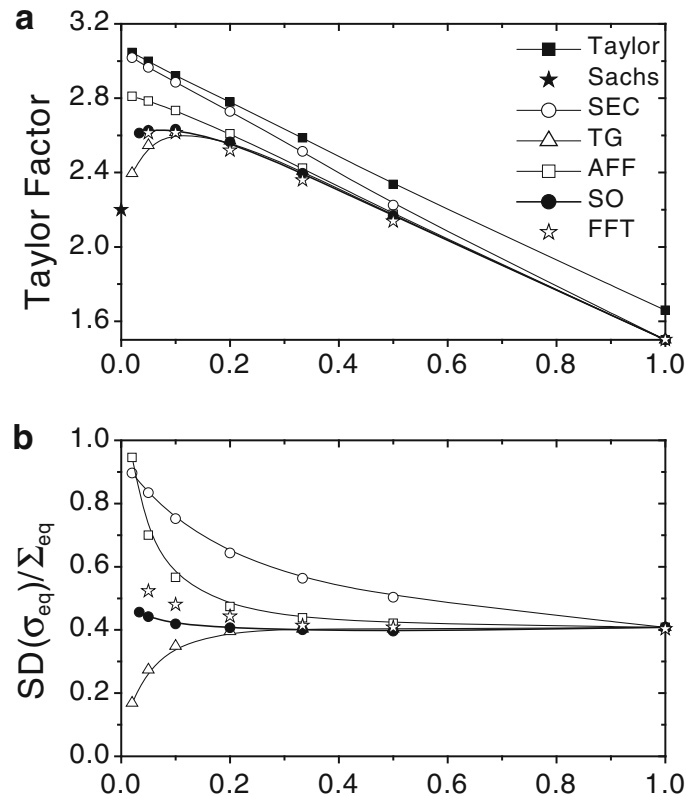

Fig. 3 (a) Average Taylor factor and normalized overall (b) stress and (c) strain standard deviations vs. rate-sensitivity, for a random fcc polycrystal under uniaxial tension, calculated with the different SC approaches (lines + symbols), and "exact" values (stars) from ensemble averages of FFT-based solutions (Lebensohn et al. 2007)

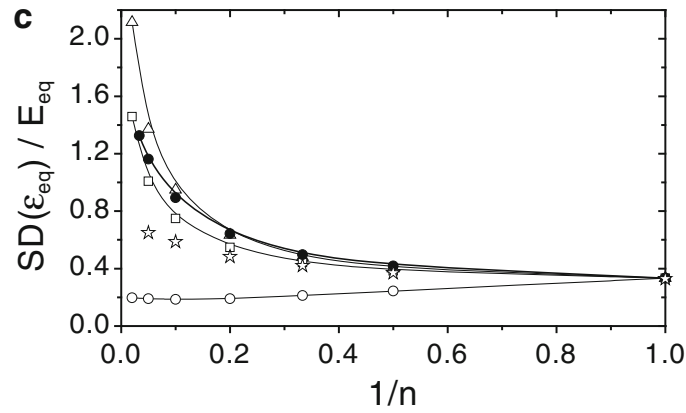


factor vs. rate-sensitivity $(1 / n)$ curves, for a random fcc polycrystal under uniaxial tension. The Taylor factor was calculated as $\Sigma_{\text {eq }}^{\text {ref }} / \tau_{\mathrm{o}}$, where $\tau_{\mathrm{o}}$ is the threshold stress of the $(111)<110>$ slip systems, and $\Sigma_{\text {eq }}^{\text {ref }}$ is the macroscopic equivalent stress corresponding to an applied uniaxial strain-rate with a Von Mises equivalent value $\dot{E}_{\text {eq }}^{\text {ref }}=1$. The curves in Fig. 3 correspond to the Taylor model, the different firstorder SC approximations, and the second-order procedure. The solid star indicates the rate-insensitive Sachs estimate. The open stars correspond to the "exact" solution, obtained from ensemble averages of FFT-based full-field solutions performed on random polycrystals. These ensemble averages were calculated over the outcomes of "numerical experiments" performed on 100 specimens generated alike, i.e., by random assignation of orientations to a given array of grains, but which differ at microlevel due to the inherent stochastic character of such generation procedure. To obtain the results that follow, we have considered periodic 3D polycrystals consisting of $8 \times 8 \times 8=512$ cubic grains with randomly chosen orientations. These unit cells were in turn discretized using a $64 \times 64 \times 64$ Fourier grid, resulting in $8 \times 8 \times 8=512$ Fourier points per grain. The averages over a sufficiently large number of configurations should give the effective properties of a polycrystal with random microstructure. It should be noted that the microstructures of these polycrystals generated for ensemble averaging are random only in a restricted sense, since the grain orientations were chosen randomly but the morphology was set a priori to be equiaxed. The generation of fully random microstructures would require grains with both random orientation and morphology (Kanit et al. 2003). However, for our purposes, the above restricted random procedure allows us to reduce the number of configurations needed to obtain an isotropic ensemble response.

From the comparison between the different mean-field and the full-field estimates, it can be observed that: (1) the Taylor approach gives the stiffest response, consistent with the upper-bound character of this model; (2) all the SC estimates coincide for $n=1$, i.e., the linear SC case; (3) in the rate-insensitive limit, the secant and tangent models tend to the upper and lower bounds, respectively, while the affine and second-order approximations remain intermediate with respect to the bounds; (4) except for the tangent model for $n>10$, the second-order procedure gives the lowest Taylor factor among the SC approaches. This softer macroscopic response (i.e., a lower stress is needed to induce a given strain-rate) is a consequence of the softer behavior at grain level in the linear comparison polycrystal that results when the average field fluctuations are considered for the determination of the linearized behavior of the SR grains; (5) the best match with the exact solutions (at least for rate-sensitivity exponents up to 20 , i.e., the highest value we were able to use in the full-field computations, without losing accuracy) corresponds to the second-order estimates.

Concerning the overall heterogeneity of the mechanical fields, reflected in the standard deviations of the equivalent magnitudes over the whole polycrystal (55-56), the SC predictions (including the second-order approximation) are less accurate. Figure 3b, c shows these overall SDs (normalized, for an unbiased comparison, by the corresponding effective magnitudes) as a function of the ratesensitivity. It can be observed that: (1) at high nonlinearities, only the SC models 
that do not tend to the bounds in the rate-insensitive limit (i.e., AFF and SO) show the expected increases in both stress and strain-rate heterogeneity. In the TG case, the stress heterogeneity decreases as the rate heterogeneity increases, while the SEC approach predicts the opposite trend; (2) both the AFF and SO approximations overestimate the strain heterogeneity; (3) the SO gives the best match with the full-field predictions for the stress heterogeneity, although it remains below the exact solution. In connection with the $\mathrm{SO}$ estimates, the use of the field fluctuations in the linear comparison material to estimate the corresponding fluctuations in the VP polycrystal has recently been shown (Idiart and Ponte Castañeda 2007) to be inconsistent. In fact, improved estimates can be generated by taking into account certain correction terms that are associated with the lack of full stationarity of these estimates with respect to the reference stresses. Still, the SC methods would not be expected to yield accurate estimates for the higher-order statistics of the fields, which become increasingly more sensitive to the details of the microstructure as the order increases. For example, the third-order moments, which contain information on the asymmetry of the distributions, are likely to become relatively important in low rate-sensitivity materials (Moulinec and Suquet 2003), since the strain tends to localize in deformation bands inside or across grains.

The next example concerns predictions of the effective behavior of random aggregates of grains with less than five linearly independent soft slip systems (Lebensohn et al. 2007). In this case, we analyze the dependence with the local contrast $\mathrm{C}$, given by the ratio between the critical stresses associated with the hard and the soft slip modes. Figure 4 shows the predicted effective stress, relative to the threshold stress of the soft slip systems $\Sigma_{\text {eq }}^{\text {ref }} / \tau_{\mathrm{o}}^{\text {soft }}$ (where $\Sigma_{\text {eq }}^{\text {ref }}$ corresponds to an applied uniaxial strain-rate, with a Von Mises equivalent $\dot{E}_{\mathrm{eq}}^{\text {ref }}=1$ ), as a function the local contrast $\mathrm{C}$, predicted by different homogenization approaches, and by averaging 100 FFT-based solutions, for the following cases:

1. A random hcp aggregate with four linearly independent soft slip systems, given by a suitable combination of $\{1010\}<1120>$ prismatic (pr) slip, and $(0001)<1120>$ basal (bas) slip (such that $\tau_{\mathrm{o}}^{\mathrm{soft}}=\tau_{\mathrm{o}}^{\mathrm{pr}}=\tau_{\mathrm{o}}^{\mathrm{bas}}$ ). The hard slip mode is $\{1011\}<1123>$ pyramidal- $<c+a>$ of the first-type (pyr1), and the contrast parameter is therefore given by $C=\tau_{\mathrm{o}}^{\mathrm{pyr} 1} / \tau_{\mathrm{o}}^{\mathrm{pr}}=\tau_{\mathrm{o}}^{\mathrm{pyr} 1} / \tau_{\mathrm{o}}^{\mathrm{bas}}$. Assuming a rate-sensitivity exponent $n=4$ and a $c / a$ ratio of 1.587 makes the above material model appropriate for a $\mathrm{Ti}$ aggregate deforming at elevated temperatures (Semiatin and Bieler 2001)

2. A random orthorhombic aggregate, with three linearly independent soft slip systems, given by a suitable combination of (010)[100], (001)[100], (010)[001], (100)[001]. The hard mode, which closes the single crystal yield surface, is assumed to be $\{111\}<110>$. All the soft systems were assumed to have the same threshold stress $\tau_{\mathrm{o}}^{\text {soft }}$, resulting in a contrast parameter $C=\tau_{\mathrm{o}}^{\{111\}} / \tau_{\mathrm{o}}^{\text {soft }}$. With a rate-sensitivity exponent $n=4$ and $b / a$ and $c / a$ ratios of 2.122 and 1.245, respectively, this material model is consistent with the behavior of an olivine polycrystal, deforming under conditions found in the Earth's upper mantle (Wenk and Tomé 1999; Castelnau et al. 2008). 
hcp, $\mathrm{n}=4,4$ independent soft slip systems (Ti)
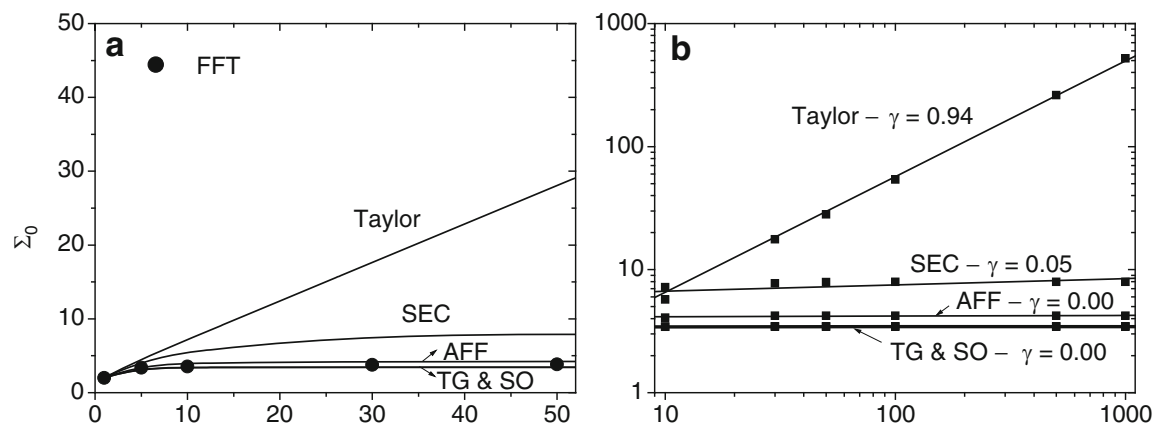

orthorhombic, $n=4,3$ independent soft slip systems (olivine)
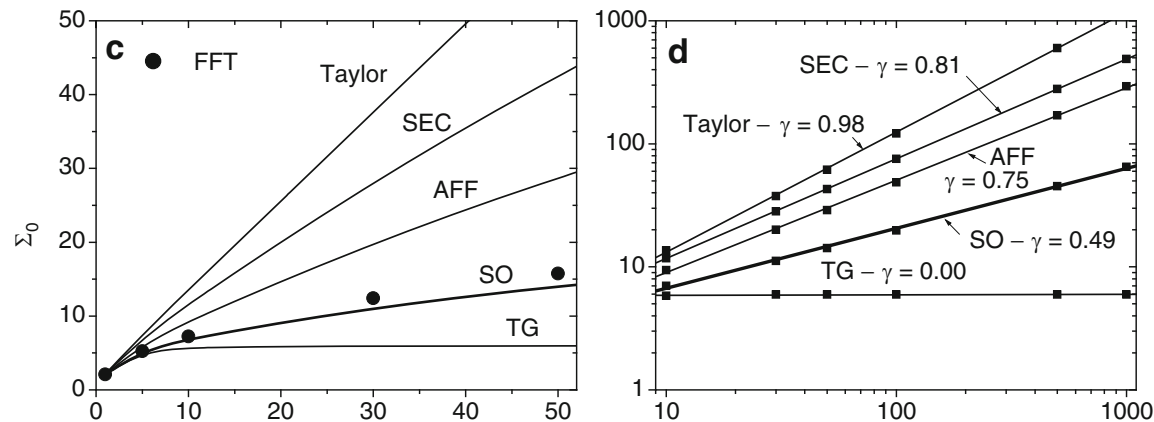

hcp, $\mathrm{n}=3,2$ independent soft slip systems (ice)
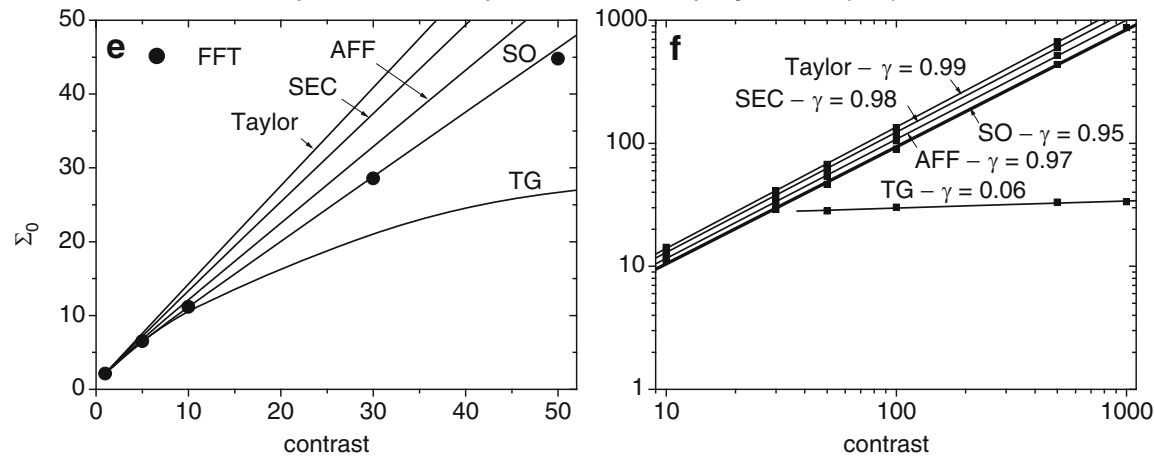

Fig. 4 Plots of reference stress vs. contrast, for random polycrystals with different number of independent soft slip systems, obtained with different SC approaches (lines) and from ensemble averages of FFT-based solution (symbols) for $(\mathbf{a}, \mathbf{b})$ four, $(\mathbf{c}, \mathbf{d})$ three $(\mathbf{e}, \mathbf{f})$ two independent soft slip systems, obtained with different SC approaches (lines) and from ensemble averages of FFT-based solution (symbols). Left column: linear scale plots, up to a contrast of 50. Right column: log-log plots, up to a contrast of 1,000 . The value of $\gamma$ corresponds to the slope of the logarithmic line (Lebensohn et al. 2007) 
3. A random hcp aggregate with two linearly independent soft systems, corresponding to $\{0001\}<1120>$ basal slip (i.e. $\tau_{\mathrm{o}}^{\text {soft }}=\tau_{\mathrm{o}}^{\mathrm{bas}}$ ). The hard slip modes are the $\{1122\}<1123>$ pyramidal $-<\mathrm{c}+\mathrm{a}>$ of the second-type (pyr2), and the contrast parameter is given by $C=\tau_{\mathrm{o}}^{\mathrm{pr}} / \tau_{\mathrm{o}}^{\mathrm{bas}}=\tau_{\mathrm{o}}^{\mathrm{pyr} 2} / \tau_{\mathrm{o}}^{\mathrm{bas}}$. Assuming a ratesensitivity exponent $n=3$ and a $c / a$ ratio of 1.629 , this material model is relevant for ice polycrystals deforming under conditions found in glaciers (Castelnau et al. 1996).

Figure 4a,c,e shows the curves (plotted in linear scale) of reference stress (i.e., $\Sigma_{\mathrm{o}}=\Sigma_{\text {eq }}^{\mathrm{ref}} / \tau_{\mathrm{o}}^{\mathrm{soft}}$, for $\dot{E}_{\mathrm{eq}}^{\mathrm{ref}}=1$ ) vs. contrast C, predicted with the different SC approximations, the Taylor model, and the full-field FFT-based solution, for C up to 50. The agreement between the SO estimates and the exact solutions is apparent. Figure $4 b$, d, f shows log-log plots of the effective stress obtained with the different homogenization models, for contrasts up to 1,000 , with the corresponding regression lines superimposed. It is evident that the results for all models can be described by scaling laws of the form $\Sigma_{\mathrm{o}} \sim C^{\gamma}$ (Nebozhyn et al. 2000). In every case analyzed ( $i=2,3$, and 4 , where $i$ is the number of linearly independent soft systems) $\gamma \cong 1$ for the Taylor model and $\gamma \cong 0$ for the tangent SC approach (note that the latter exponent also corresponds to the lower-bound Sachs model), while the secant, affine, and second-order SC models give different exponents, depending on the value of $i$. Interestingly, the exponents corresponding to the second-order approach follow the relation proposed by Nebozhyn et al. (2000): $\gamma \cong(4-i) / 2$, in the context of Ponte Castañeda's (1991) variational approach. The asymptotic trend to the lower-bound that the tangent SC approach exhibits when the contrast increases due to the increase of the exponent $n$ is also obtained when the heterogeneity increases due to local anisotropy, even for relatively low values of $n$. This observation sheds light on why the tangent SC approach has been favored to predict mechanical behavior of low-symmetry materials, which have "open" single crystal yield surfaces with three or less independent deformation systems. In such cases, the tangent SC approach allows accommodation of the local deformation with the available slip systems, without need of "artificial" systems to close the single crystal yield surface. While these artificial hard systems make a very small contribution to strain, they have a strong influence on the predicted macroscopic behavior (effective viscosity) in these low-symmetry systems, unless a saturated behavior, like the one displayed by the tangent predictions in Fig. 4, is obtained.

\subsection{Overall Texture Development Predictions Using Mean-Field Approaches}

Almost $100 \%$ of plastic deformation in the ice single crystals is carried by basal dislocations. Since basal slip provides only two independent slip systems, the prediction of texture development of polycrystalline ice is a challenging problem that allows us to discriminate among the different SC approaches. Moreover, an 
understanding of the deformation mechanisms and the microstructural evolution of ice deforming in compression is relevant in glaciology, since compression (together with shear) is one of the main deformation modes of glaciers. In what follows, we will use the basal texture factor along the axial direction to characterize the evolving texture of ice in compression. The basal texture factor is defined as the weighted average of the projections of the $c$-axis along the axial direction, i.e., $\left\langle\cos ^{2} \alpha^{(r)}\right\rangle$, where $\alpha^{(r)}$ is the angle between the basal pole of SR grain $(r)$ and the axial compression direction.

In fact, the stiff Taylor and SC secant models are not suitable to simulate plastic deformation of polycrystalline ice because the strong constraints that these models impose upon strain are incompatible with the shortage of independent slip systems in ice. On the other hand, the compression textures of ice typically exhibit a strong basal pole component aligned with the axial direction (Castelnau et al. 1996). The formation of this component is related to the crystallographic plastic rotations associated with basal slip. However, as the basal poles become more aligned with the axial direction, the basal systems become unfavorably oriented to accommodate deformation. Therefore, at large strains, even a "soft" first-order approximation like the tangent $\mathrm{SC}$ fails in reproducing the observed texture with only basal slip activity (Castelnau et al. 1996). Up to now, the Sachs model (which completely disregards strain compatibility) has been the only approach able to give a reasonably effective behavior with predominant basal slip at large strains, when the basal texture along the compressive direction becomes very strong.

Figure 5 shows the compression texture evolution (in terms of the basal texture factor), effective stress, relative basal activity, and average number of active slip systems (AVACS) per grain, for the case of an initially random ice polycrystal (Lebensohn et al. 2007). Results were obtained using the TG, AFF, and SO approaches, under the assumption of $n=3$ and $\tau_{\mathrm{o}}^{\mathrm{pr}}=20 \times \tau_{\mathrm{o}}^{\mathrm{bas}}$ and $\tau_{\mathrm{o}}^{\mathrm{pyr} 2}=200 \times \tau_{\mathrm{o}}^{\mathrm{bas}}$ (Castelnau et al. 1996), with no strain-hardening, up to a compressive strain of 1.5.

As expected, all models predict a prevalence of basal slip, with a consequent increase of the basal texture factor along the axial direction, and a progressive geometric hardening. While the alignment of basal poles along the compression direction predicted by all three models is similar, they differ in other indicators. At around 0.8 strain, the tangent predictions show a sudden drop in the basal activity, together with a rapid increase in the effective stress and in the number of active deformation systems, which indicates that the strain accommodation starts requiring the activation of the 200 times harder pyramidal systems. In other words, under the tangent SC approach, the basal slip by itself is not enough to accommodate the compressive deformation when the basal poles become strongly aligned with the compression direction.

The SO and AFF models, on the other hand, do a better job at accommodating large strain mostly with basal slip. The SO results, however, are superior to the AFF results in this respect. This superior performance of the second-order SC approximation can be explained in terms of its intrinsic adaptability to microstructural changes. Figure 6 shows the evolution (as predicted with the SO formulation) of the normalized standard deviations of the equivalent stress and strain rate over the whole 
Fig. 5 Simulation of compression of an ice polycrystal. (a) Basal texture factor along the compression direction, (b) effective stress, (c) relative basal activity, and (d) the average number of active slip systems per grain, as predicted with the tangent (TG), affine (AFF), and second-order (SO) SC approaches (Lebensohn et al. 2007)

Fig. 6 Evolution of the normalized overall standard deviations of the equivalent stress and strain-rate, as predicted with the second-order formulation, for the case of ice in compression (Lebensohn et al. 2007)
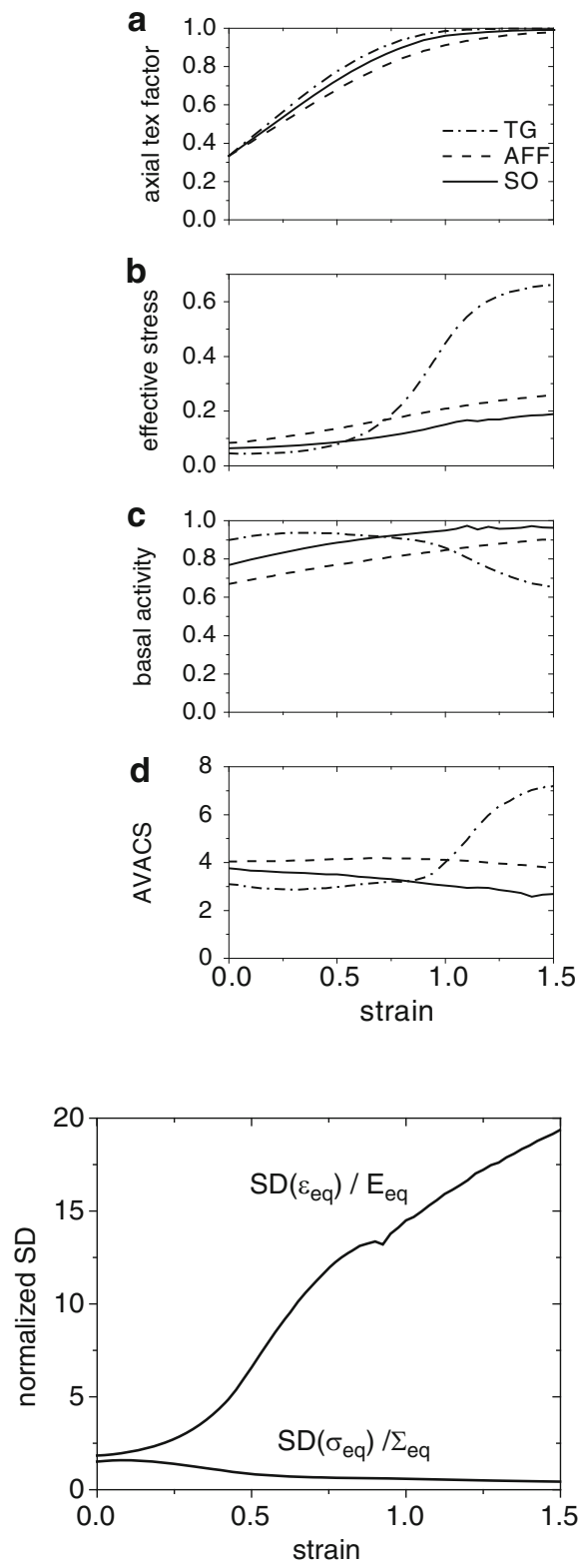

polycrystal, defined by (55-56). Note that the above magnitudes are indicators not only of intergranular but also of intragranular heterogeneity (as a matter of fact, these average scalar magnitudes reflect the collective contribution of every component of the fluctuation tensors in each SR grain). Evidently, as the basal texture concentrates along the axial direction, the stress becomes more uniform and the 
strain-rate becomes more heterogeneous. This trend toward a uniform stress state obviously indicates a trend toward the Sachs condition. Therefore, given that the aforementioned local fluctuation information is contained in the second-order linearization, the SO results approach the lower-bound as deformation proceeds, allowing a substantial accommodation of deformation by basal slip at those large strains.

\subsection{Local Texture Development Predictions Using the FFT-Based Full-Field Approach}

Owing to its image-processing lineage, the FFT-based formulation is particularly suitable for use with direct input from actual images of the material, e.g., optical or scanning electron microscopy (SEM) images that show the phase distribution in the case of composites (Moulinec and Suquet 1998), or orientation images in the case of polycrystals (Lebensohn et al. 2008). The latter will be used here for a quantitative study of the average orientations and intragranular misorientations developed in a $\mathrm{Cu}$ polycrystal deformed in tension. Electron back-scattering diffraction (EBSD)-based OIM was used to characterize the local orientations measured in an area of about $500 \times 500 \mu \mathrm{m}$, located on one of the flat surfaces of a recrystallized copper sample. The spatial resolution (given by the distance between two consecutive pixels) was $2 \mu \mathrm{m}$ in each direction. Two OIM images were taken, one from the undeformed sample, and another after $11 \%$ tensile strain along the $y$-direction (deformation was carried out at room temperature). The scanned area of the deformed sample $(332 \times 445$ pixels $)$ was larger than that of the initial microstructure $(274 \times 339$ pixels), and contained more orientations (2,429 vs. 1,585 grains). This allowed us to register the images, and thus to identify the ID numbers given by the OIM software to each individual grain. Once the two images were appropriately registered, a correlation table allowed us to identify the ID numbers of the grains with largest areas, in the pre- and postdeformation images, for further comparisons.

Next, a 2D $256 \times 256$ image, containing information on the local (pixel by pixel) crystallographic orientation and a total of 1,124 grains, was cropped from the original OIM image, obtained from the free surface of the undeformed $\mathrm{Cu}$ sample, consisting originally of $274 \times 339$ pixels and 1,585 grains. The average grain size (in units of length) of the cropped image, which can be roughly estimated, is: $\sqrt{256 \times 256 / 1124} \times 2 \mu \mathrm{m}=7.64 \times 2 \mu \mathrm{m}=15.28 \mu \mathrm{m}$. Note, however, that, since the grains in the 2D image are not necessarily sliced across their largest extent as projected onto the observation plane, the above grain size is a low estimate of the true grain size in $3 \mathrm{D}$.

Since the actual 3D microstructure of the bulk of the sample was not known, a 3D unit cell was built assuming a randomly generated distribution of bulk grains underneath the measured surface grains (i.e., a "3D substrate"), having same average grain size and overall crystallographic orientation distribution as the surface grains. For this, a 3D Voronoi was generated (note that since the FFT-based calculation requires a discrete description of the microstructure on a regularly spaced grid, the procedure is simpler than in the case of having to determine the exact position of 
the boundaries between Voronoi cells in a continuum), as follows: (1) the number of Fourier points in the third dimension (z-direction) was chosen to be 32, resulting in a unit cell of $256 \times 256 \times 32=2,097,152$ Fourier points. Note that this choice gives, in average, about four grains along third dimension; (2) the number of grains of the Voronoi structure was calculated as $2,097,152 /(7,64)^{3}=4,703$; (3) then, 4,703 points were randomly distributed in a $3 \mathrm{D}$ unit cell. This Poisson distribution of points constitutes the nuclei of the random grains; (4) the sides of the unit cell were divided into equispaced $256 \times 256 \times 32$ Fourier points, or voxels. Each Fourier point was assigned to its nearest nucleus (accounting for periodic boundary conditions across the unit cell limits), determining 4,703 different domains (grains). Next, the measured 2D and the numerically generated 3D microstructures were merged as follows. First, every 3D grain having a voxel on the first z-layer was removed, and every voxel corresponding to these removed grains was assigned with the crystallographic orientation of the pixel of the 2D OIM image having the same $x$ - and $y$-coordinates. These replacements determined a structure of "extruded" (columnar) grains of variable depth in the $z$-direction (from one to several layers), with its first ("surface") layer having the same topology as the OIM image, lying on a 3D substrate. The number of grains of this intermediate configuration decreased to 3,965 grains. Subsequently, to obtain more realistic grain shapes, especially in the transition zone between the columnar grains and the 3D substrate, the microstructure was "annealed" using a standard 3D Monte Carlo (MC) grain growth model with isotropic boundary properties (Rollett and Manohar 2004). The voxels in the surface layer that corresponded to the measured OIM scan (reproduced on the bottom layer, with periodic boundary conditions) were fixed and not allowed to evolve. All other parts of the microstructure were allowed to evolve with the result that grain boundaries moved to minimize their areas. The annealing was run for 1,000,000 MC steps, at which time evolution had essentially ceased because of the pinning effect of the surface layers. The number of grains was further decreased to 3,697 in the final annealed microstructure.

As already pointed out, after carrying out this numerical treatment of the unit cell's microstructure, the first layer of the resulting representative volume element turned out to have the exact same topology as the OIM image. However, without any further manipulation of this configuration, the measured "surface" grains would become bulk grains, upon the imposition of periodic boundary conditions across the unit cell. Therefore, to reproduce the actual free surface condition on the measured grains, the bottom five $z$-layers ( $z$-layers 28-32) of Fourier points were replaced by a "buffer zone," or "gas phase," with infinite compliance (i.e., identically zero local stress). Such gas phase allowed us to consider the presence of surface grains (corresponding precisely to the grains whose local orientations were actually measured by OIM) while keeping, at the same time, the periodicity across the unit cell (this buffer "disconnects" the surface from the bottom of the periodic repetition of the unit cell, located immediately above). A similar technique was used in phase field simulations of microstructure evolution in thin films (Hu and Chen 2004). The resulting configuration of the 3D unit cell, including the zero-stress buffer zone, is shown schematically in Fig. 7 (Lebensohn et al. 2008). In the next section, we show 


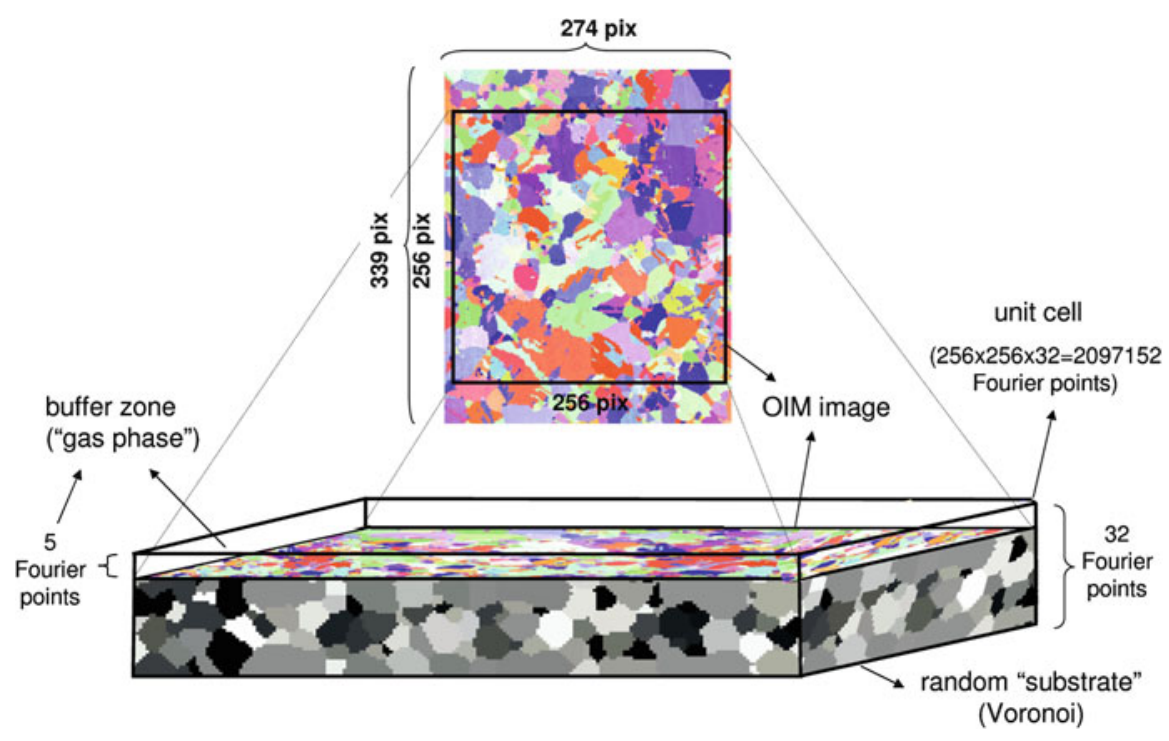

Fig. 7 Schematic representation of the 3D unit cell used in the FFT-based simulations of local orientation and misorientation evolution, with direct input from OIM images (Lebensohn et al. 2008)

and compare results of both unit cell configurations, i.e., the original one resulting from the merging of the OIM and Voronoi structures plus the MC annealing, with no buffer zone (amounting to neglect the surface character of the grains whose orientations were measured by OIM), and the one including the gas phase, for a direct comparison with the OIM measurements.

FFT-based simulations of the tensile deformation of the polycrystalline copper sample were performed using the two above-described unit cells (with and without the buffer zone). The rate-sensitive crystal plasticity relation (1) was used as the local constitutive relation, assuming glide on the twelve $(111)<110>$ systems as the active slip mode, and a viscoplastic exponent $n=20$. The initial distribution of critically resolved shear stresses was assumed to be uniform. The extended Voce law hardening parameters (74), adjusted to match the experimental macroscopic stressstrain curve measured during the tensile deformation of the copper sample, were: $\tau_{\mathrm{oo}}^{s}=11 \mathrm{MPa}, \quad \tau_{1}^{s}=15.5 \mathrm{MPa}, \quad \theta_{0}^{s}=430 \mathrm{MPa}, \quad \theta_{1}^{s}=110 \mathrm{MPa}, \quad(s=1,12) \quad$ and $h^{s s^{\prime}}=1$, for all $s s^{\prime}$.

Figure 8 a shows the registered initial and 11\% strain OIM images (the latter is shown already cropped), measured on the surface of the copper sample (Lebensohn et al. 2008). The postdeformation image clearly indicates the development of intragranular misorientations, in terms of noticeable color grades inside several grains. The location and number of pixels of the 13 largest ("marked") grains are shown in Fig. 8b. The initial orientations of the marked grains can be seen in Fig. 9, in an inverse pole figure representation. Figure 9 also shows the trajectories of the mean orientations of these marked grains (except for grain \#5, which is very close 


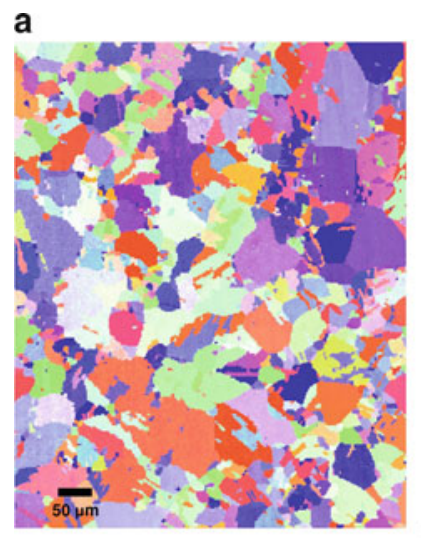

b

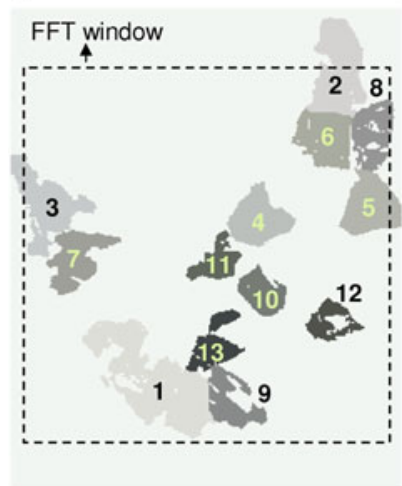

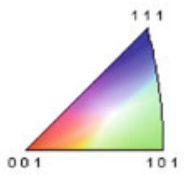
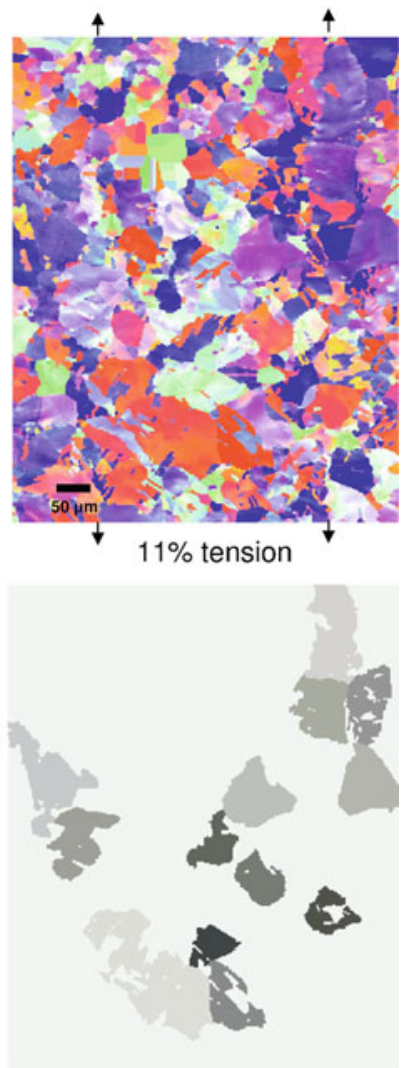

Fig. 8 (a) Registered OIM images of the copper polycrystal before deformation and after $11 \%$ tensile strain. (b) Location and morphology of the 13 largest grains, before and after deformation. The "FFT window" shows the $256 \times 256$ pixel region that was actually used to construct the unit cell (Lebensohn et al. 2008)

Fig. 9 Inverse pole figure of the measured initial orientation and the final average orientation of the largest grains, and trajectories predicted with the FFT-based approach (Lebensohn et al. 2008)

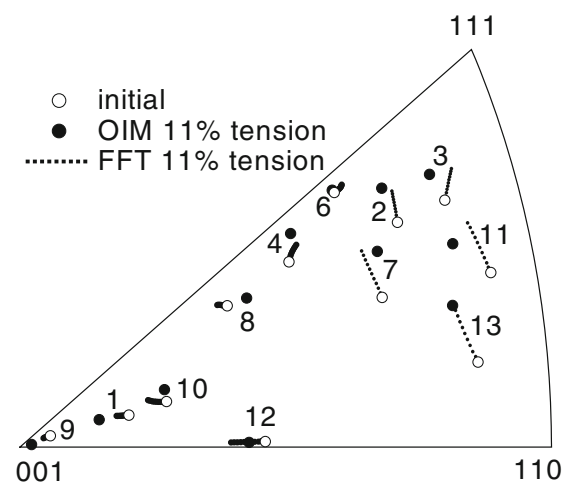


and behaves very similarly to grain \#6, and was not plotted for sake of clarity), as predicted by the FFT-based model (with buffer zone). The small crosses defining these trajectories were obtained in increments of $1 \%$ overall plastic strain. The actual final average orientations, measured with OIM, are shown as well. In the region close to the $<001>$-corner, grains \#1, \#9, and \#12 rotate toward the stable orientation $<001>$, a trend that is predicted, at least qualitatively, by the model. Grains with initial orientations close to the upper half of the $<001>-<111>-$ line (\#4, \#5, and \#6) exhibit rotations along this line toward the other stable orientation, i.e., $<111>$ (also well reproduced by the model). The grains starting near the $<110>$-corner (\#11 and \#13), or in intermediate orientations between $<110>$ and the midsection of the $<001>-<111>$-line (\#2, \#3 and \#7) rotate toward this line, presumably in their way toward the stable orientation $<111>$. The total rotations of these grains are the largest. All these features are acceptably reproduced by our simulations, except for the reorientation of grain \#3, which is predicted to be directly toward $<111>$. Finally, the trends of grain \#10, and especially grain \#8, which starts close a well-known transition point on the $<001>-<111>$-line, close to $<113>$ (Chin et al. 1967), are not adequately reproduced by the model. It is also interesting to compare (while keeping in mind the obvious differences, i.e., $\mathrm{Cu}$ vs. $\mathrm{Al}, 2 \mathrm{D}$ vs. 3D) the above trends and the 3D X-ray diffraction characterization done by Winther et al. (2004) of the rotations of bulk grains of an Al polycrystal deformed in tension. The rotations measured on $\mathrm{Cu}$ surface grains (and well predicted by our model) with initial orientations belonging to three of the four "regions" characterized by Winther et al. (2004) (i.e., region 1: close to $<111>$, region 2: near the upper half of the $<110>-<111>$-line, and region 4 : near the $<100>$-corner) are also in acceptable agreement with the observations of Winther et al. (2004). No grains with orientations in Winther et al.'s region 3 (close to $<221>$ ) were large enough in the $\mathrm{Cu}$ sample to be used for this analysis.

Although interesting, the above-described reasonable agreement between the measured and predicted average orientation evolution is not surprising, since almost every (either full-field or mean-field) model based on crystal plasticity qualitatively predicts the development of two stable texture components in $<001>$ and $<111>$ in fcc materials deformed under tension, e.g., see comparisons of the above-mentioned measurements with corresponding Taylor and self-consistent predictions in Winther et al. (2004). A much less investigated aspect of the local texture evolution of these materials is reported in Table 2, which shows the comparison between the measured and the predicted values (in degrees) of the average misorientations (defined as the average over every pixel belonging to a given grain with respect to the average orientation of that grain, a quantity that can be readily calculated using quaternion algebra) inside the marked grains (Lebensohn et al. 2008). Together with the size (in pixels) of the grains, two predicted values are reported for each grain: the fourth column shows the predictions obtained using the unit cell shown in Fig. 7, i.e., including the buffer zone, therefore considering the effect of measuring misorientations in surface grains. The fifth column displays the predictions obtained for a unit cell without the buffer zone, giving an idea of the misorientation values that would be measured if the grains were bulk. Evidently, the proper consideration in 
Table 2 Area (in pixels) and average misorientation of the 13 largest grains after $11 \%$ tensile strain, measured by OIM and predicted with the FFT-based approach, with and without buffer zone (Lebensohn et al. 2008)

\begin{tabular}{lllll}
\hline & $\begin{array}{c}\text { Number } \\
\text { Grain no. }\end{array}$ & $\begin{array}{l}\text { Average misorientation } \\
\text { of pixels }\end{array}$ & $\begin{array}{l}\text { Average misorientation 11\% tension (deg) } \\
\text { FFT 11\% tension (deg) }\end{array}$ & $\begin{array}{l}\text { Average misorientation } \\
\text { (no buffer) (deg) }\end{array}$ \\
\hline 1 & 3,625 & 2.89 & 2.18 & 1.81 \\
2 & 2,019 & 2.52 & 2.05 & 1.64 \\
3 & 1,842 & 2.92 & 2.97 & 2.25 \\
4 & 1,479 & 2.86 & 2.33 & 2.27 \\
5 & 1,466 & 2.26 & 2.35 & 1.76 \\
6 & 1,380 & 3.14 & 2.70 & 1.91 \\
7 & 1,331 & 3.37 & 3.06 & 2.89 \\
8 & 1,113 & 3.21 & 2.62 & 2.12 \\
9 & 955 & 2.65 & 2.80 & 2.22 \\
10 & 830 & 2.92 & 2.37 & 1.81 \\
11 & 692 & 2.22 & 2.79 & 1.99 \\
12 & 639 & 4.33 & 3.36 & 2.39 \\
13 & 596 & 3.09 & 3.26 & 2.83 \\
\hline
\end{tabular}

the model of the surface character of the grains, whose average misorientations were measured by OIM, leads to a good agreement with the corresponding experimental values. On the other hand, the artificial assumption of the bulk character of these grains tends to underestimate the actual average misorientations of surface grains. The reason why the predictions obtained under the bulk assumption fall short is related to this being a different configuration, compared with the actual traction-free boundary conditions imposed on the surface grains.

Another interesting observation is that except for grain \#12, which exhibits the largest average misorientation, the initial orientations of other grains with measured misorientations larger than $3.0^{\circ}(\# 6, \# 7, \# 8$, and \#13) lie in a region of the stereographic triangle spanning from $<110>$ toward the midsection (from $1 / 3$ to $2 / 3$ ) of the $<001>-<111>-$-line. While in the case of grain \#12, the high average misorientation seems to be related to its particular morphology (note in Fig. 8b that this grain has a large "hole" in its center, filled by another grain with a completely different orientation, a configuration that may determine a relative "disconnection" between different parts of grain \#12); for the rest of the grains, their initial orientations belonging to the above region may be related to their relatively large average misorientation.

To elucidate whether this orientation dependence does exist (and if our model is capable of reproducing it), we investigated the behavior of a larger number of representative grains. Figure 10 shows the average orientations (given by each pole projected in the inverse pole figure) and the average misorientations (given by the different symbols used) of the largest 306 grains, as measured by OIM and predicted with the FFT-based approach, after 11\% tensile strain (Lebensohn et al. 2008). The misorientation values of the grains were grouped into six bins of equal size, and different symbols were assigned to each bin. The first observation is that, as expected, 

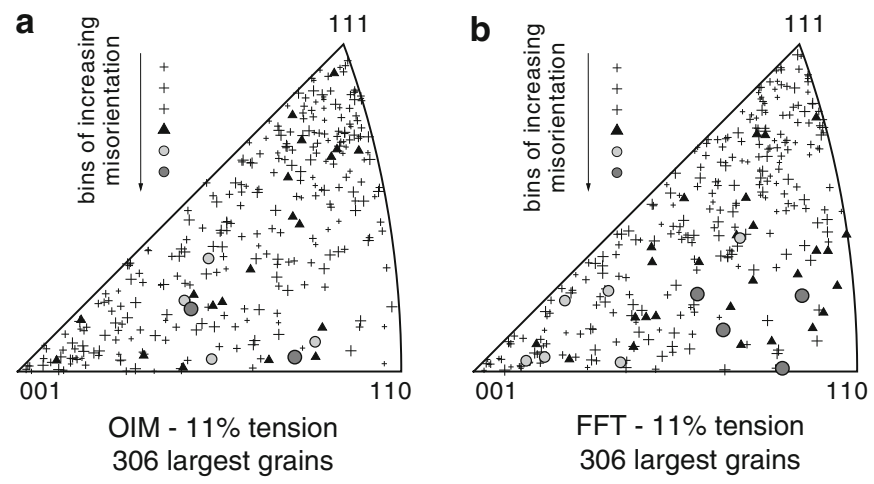

Fig. 10 Inverse pole figures of the average orientations and misorientations of the 306 largest grains after $11 \%$ tensile strain, (a) measured by OIM, and (b) predicted with the FFT-based approach. The misorientations were grouped in bins of equal size, and different symbols were assigned to each bin (Lebensohn et al. 2008)

after $11 \%$ tension, there is already a mild but noticeable trend of large grains to rotate toward one of the stable $<001>$ and $<111>$ orientations (the region near $<110>$ is mildly depleted of orientations). Moreover, it is evident (from both the experiments and the simulations) that most of the grains with the highest average misorientation are grains transitioning from their initial orientation near $<110\rangle$ toward the stable orientations. This observation can be explained in the following terms: depending on their initial orientation, the grains of an fcc polycrystal in tension are attracted toward one of the two stable orientations, i.e., $<001>$ or $<111>$ (the latter, directly or via the $<001>-<111>-$ line). Grains with orientations in a region of the orientation space, spanning from near $<110>$ toward the midsection of the $<001>-<111>-$ line, can be pulled simultaneously toward both stable orientations. In this case, the instability of the initial grain orientation and the contribution of interactions with neighbor grains may define the preference of different portions of these "indecisive" grains to rotate toward different stable orientations. This conflicting attraction toward two completely different orientations may be accommodated by the development of relatively higher misorientations between different grain's subdomains. This corresponds to the transition band concept documented by Dillamore et al. (1972) and Dillamore and Katoh (1974) in polycrystals. Note also that this orientation split is also observed in deformed single crystal with initial unstable orientations [thus implying no grain interaction effect (Becker et al. 1991)].

\section{Conclusions}

In this chapter, we have thoroughly reviewed recently proposed some novel crystal plasticity-based methods for the prediction of microstructure-property relations in polycrystalline aggregates. The first method is of the mean-field type and is known 
as the second-order VPSC theory, while the second is a full-field method and is known as the FFT-based formulation. We have comprehensively presented the equations and assumptions underlying both formulations, using a unified notation and pointing out their similarities and differences. Mean-field approaches are in general much more efficient than full-field computations. However, models like the secondorder VPSC formulation, which incorporates more statistical information, require more complex and numerically demanding algorithms, but still are much faster than full-field approaches.

Concerning the mean-field theories, we have carried out detailed comparisons of the different self-consistent approximations for viscoplastic polycrystals. We have also discussed the numerical implementation of the different SC approaches in the VPSC code, together with results obtained using different linearization strategies. The comparison of the effective behavior of model material systems predicted by different SC approaches has shown that the second-order SC predictions are in better agreement with the ensemble averages of FFT-based full-field solutions. The latter is especially true in the cases of highly heterogeneous materials (due to a strong nonlinearity or local anisotropy), a case in which the gap between the Taylor and the Sachs bounds is large. With regards to applications of the second-order SC approximation, we have studied the texture evolution of polycrystalline ice (a material characterized by a strong local anisotropy, due a strong contrast of plastic properties at single crystal level) deformed in compression, to illustrate the flexibility of the second-order formulation to handle these highly anisotropic problems.

The FFT-based full-field formulation for plastically deforming polycrytals has been conceived as a very efficient alternative to crystal-plasticity FE methods. In this work, FFT-based computations were first applied to the antiplane deformation of isotropic, linearly viscous 2D polycrystals. In this case, our numerical implementation was validated by comparison with analytical results for the effective and per-phase properties of such special configuration. Next, the full 3D implementation was applied to the study of the subgrain texture evolution in a copper aggregate deformed under tension. Direct input was obtained from OIM images for the construction of the representative volume element. A methodology to build such 3D unit cell, including the 2D OIM data, a 3D substrate, and the presence of a free surface, was given. The average orientations and misorientations of large grains, predicted with the FFT-based approach after $11 \%$ tensile strain, were directly compared with OIM measurements. The experimental data and the predictions showed good agreement. The orientation dependence of the average misorientations was also studied. Again, measurements and predictions showed reasonable agreement. Grains with initial orientation near $<110>$ tend to develop higher misorientations, as deformation proceeds. Attraction toward the two different stable orientations (i.e., corresponding to the alignment of the $<001>$ and the $<111>$ crystal orientations with the tensile axis) of different subdomains inside these grains, influenced by interactions with different neighbors, may be responsible for this behavior. Only full-field models such as the FFT-based formulation, which account for topological information and grain interaction in the determination of the local micromechanical fields, can capture these effects. 
Acknowledgments We wish to thank our colleagues Carlos Tomé (LANL, Los Alamos, USA), Tony Rollett (CMU, Pittsburgh, USA), Pierre Gilormini (ENSAM, Paris, France), and Pierre Suquet (LMA, Marseille, France) for fruitful discussions.

\section{References}

Acharya A, Bassani JL, Beaudoin A (2003) Geometrically necessary dislocations, hardening, and a simple gradient theory of crystal plasticity. Scr Mater 48: 167-172.

Barbe F, Decker L, Jeulin D, Cailletaud G (2001) Intergranular and intragranular behavior of polycrystalline aggregates. Part 1: F.E. model. Int J Plast 17: 513-536.

Becker R (1991) Analysis of texture evolution in channel die compression. 1. Effects of grain interaction. Acta Metall Mater 39: 1211-1230.

Becker R, Butler JF, Hu H, Lalli LA (1991). Analysis of an aluminum single-crystal with unstable initial orientation (001)[110] in channel die compression. Metall Trans A 22:45-58.

Berveiller M, Fassi-Fehri O, Hihi A (1987) The problem of 2 plastic and heterogeneous inclusions in an anisotropic medium. Int J Eng Sci 25: 691-709.

Bhattacharyya A, El-Danaf E, Kalidindi SR, Doherty RD (2001) Evolution of grain-scale microstructure during large strain simple compression of polycrystalline aluminum with quasicolumnar grains: OIM measurements and numerical simulations. Int J Plast 17: 861-883.

Bobeth M, Diener G (1987) Static elastic and thermoelastic field fluctuations in multiphase composites. J Mech Phys Solids 35:137-149.

Castelnau O, Duval P, Lebensohn RA, Canova GR (1996) Viscoplastic modelling of texture development in polycrystalline ice with a self-consistent approach: comparison with bound estimates. J Geophys Res B 101: 13851-13868.

Castelnau O, Blackman DK, Lebensohn RA, Ponte Castañeda P (2008) Micromechanical modelling of the viscoplastic behavior of olivine. J Geophys Res B 113: B09202.

Chen LQ (2004) Introduction to the phase-field method of microstructure evolution. In: Raabe D, Roters F, Barlat F, Chen LQ (eds). Continuum scale simulations of engineering materials. Wiley, Wenheim, pp. 37-51.

Cheong KS, Busso EP (2004) Discrete dislocation density modelling of single phase FCC polycrystal aggregates. Acta Mater 52: 5665-5675.

Chin GY, Mammel WL, Dolan MT (1967) Taylor's theory of texture for axisymmetric flow in body-centered cubic metals. Trans Met Soc AIME 239: 1854-1855.

deBotton G, Ponte Castañeda P (1995) Variational estimates for the creep-behavior of polycrystals. Proc R Soc Lond A 448: 121-142.

Delaire F, Raphanel JL, Rey C (2001) Plastic heterogeneities of a copper multicrystal deformed in uniaxial tension: experimental study and finite element simulations. Acta Mater 48: 1075.

Delannay L, Logé RE, Chastel Y, Signorelli JW, Van Houtte P (2003) Measurement of in-grain orientation gradients by EBSD and comparison with finite element results. Adv Eng Mater 5: 597-600.

Delannay L, Jacques PJ, Kalidindi SR (2006) Finite element modeling of crystal plasticity with grains shaped as truncated octahedrons. Int J Plast 22: 1879-1898.

Diard O, Leclercq S, Rousselier G, Cailletaud G (2005) Evaluation of finite element based analysis of 3D multicrystalline aggregates plasticity Application to crystal plasticity model identification and the study of stress and strain fields near grain boundaries. Int J Plast 21: 691.

Dillamore IL, Morris PL, Smith CJE, Hutchinson WB (1972) Transition bands and recrystallization in metals. Proc R Soc Lond A 329: 405-420.

Dillamore IL, Katoh H (1974) Mechanisms of recrystallization in cubic metals with particular reference to their orientation-dependence. Met Sci J 8: 73-83.

Dykhne AM (1970) Conductivity of a two-dimensional two-phase system. Dokl Akad Nauk SSSR 59: $110-115$. 
Hershey AV (1954) The elasticity of an isotropic aggregate of anisotropic cubic crystals. J Appl Mech 21: 236-240.

Hill R (1965) Continuum micro-mechanics of elastoplastic polycrystals. J Mech Phys Solids 13: 89 .

Hutchinson JW (1976) Bounds and self-consistent estimates for creep of polycrystalline materials. Proc R Soc Lond A 348: 101-127.

Hu SY, Chen LQ (2004) Spinodal decomposition in a film with periodically distributed interfacial dislocations. Acta Mater 52: 3069-3074.

Idiart MI, Ponte Castañeda P (2007) Field statistics in nonlinear composites. I. Theory. Proc R Soc Lond A 463: 183-202.

Idiart MI, Moulinec H, Ponte Castaneda P, Suquet P (2006) Macroscopic behavior and field fluctuations in viscoplastic composites: second-order estimates versus full-field simulations. J Mech Phys Solids 54: 1029-1063.

Kanit T, Forest S, Galliet I, Mounoury V, Jeulin D (2003) Determination of the size of the representative volume element for random composites: statistical and numerical approach. Int J Solids Struct 40: $3647-3679$.

Kreher W (1990) Residual-stresses and stored elastic energy of composites and polycrystals. J Mech Phys Solids 38: 115-128.

Lahellec N, Michel JC, Moulinec H, Suquet P (2001) Analysis of inhomogeneous materials at large strains using fast Fourier transforms. In: Miehe C (ed). IUTAM symposium on computational mechanics of solids materials. Kluwer Academic, Dordretcht, pp. 247-268.

Laws N (1973) On the thermostatics of composite materials. J Mech Phys Solids 21: 9-17.

Lebensohn RA, Tomé CN (1993) A selfconsistent approach for the simulation of plastic deformation and texture development of polycrystals: application to Zirconium alloys. Acta Metall Mater 41: 2611-2624.

Lebensohn RA, Turner PA, Signorelli JW, Canova GR, Tomé CN (1998) Calculation of intergranular stresses based on a large strain viscoplastic selfconsistent polycyrstal model. Model Simul Mater Sci Eng 6: 447-465.

Lebensohn RA (2001) N-site modelling of a 3D viscoplastic polycrystal using fast Fourier transform. Acta Mater 49: 2723-2737.

Lebensohn RA, Liu Y, Ponte Castañeda P (2004a) On the accuracy of the self-consistent approximation for polycrystals: comparison with full-field numerical simulations. Acta Mater 52: 5347-5361.

Lebensohn RA, Liu Y, Ponte Castañeda P (2004b) Macroscopic properties and field fluctuations in model power-law polycrystals: full-field solutions versus self-consistent estimates. Proc R Soc Lond A 460: 1381-1405.

Lebensohn RA, Castelnau O, Brenner R, Gilormini P (2005) Study of the antiplane deformation of linear 2-D polycrystals with different microstructures. Int J Solids Struct 42: 5441-5449

Lebensohn RA, Tomé CN, Ponte Castañeda P (2007) Self-consistent modeling of the mechanical behavior of viscoplastic polycrystals incorporating intragranular field fluctuations. Phil Mag 87: 4287-4322.

Lebensohn RA, Brenner R, Castelnau O, Rollett AD (2008) Orientation image-based micromechanical modelling of subgrain texture evolution in polycrystalline copper. Acta Mater 56: 3914-3926.

Lequeu P, Gilormini P, Montheillet F, Bacroix B, Jonas JJ (1987) Yield surfaces for textured polycrystals. 1. Crystallographic approach. Acta Metall 35: 439-451.

Liu Y, Ponte Castañeda P (2004) Second-order theory for the effective behavior and field fluctuations in viscoplastic polycrystals. J Mech Phys Solids 52: 467-495.

Lurie KA, Cherkaev AV (1984). G-closure of some particular sets of admissible material characteristics for the problem of bending of thin elastic plates. J Optim Theor Appl 42: 305-316.

Masson R, Bornert M, Suquet P, Zaoui A (2000) Affine formulation for the prediction of the effective properties of nonlinear composites and polycrystals. J Mech Phys Solids 48: 1203-1227. 
Michel JC, Moulinec H, Suquet P (2000) A computational method based on augmented Lagrangians and fast Fourier transforms for composites with high contrast. Comput Model Eng Sci 1: 79-88.

Mika DP, Dawson PR (1998) Effects of grain interaction on deformation in polycrystals. Mater Sci Eng A 257: 62-76.

Molinari A, Canova GR, Ahzi S (1987) Self consistent approach of the large deformation polycrystal viscoplasticity. Acta Metall 35: 2983-2994.

Moulinec H, Suquet P (1994) A fast numerical method for computing the linear and nonlinear mechanical properties of composites. C R Acad Sci Paris II 318: 1417-1423.

Moulinec H, Suquet P (1998) Numerical method for computing the overall response of nonlinear composites with complex microstructure. Comput Methods Appl Mech Eng 157: 69-94.

Moulinec H, Suquet P (2003) Intraphase strain heterogeneity in nonlinear composites: a computational approach. Eur J Mech Solids 22: 751-770.

Mura T (1987) Micromechanics of defects in solids. Martinus-Nijhoff Publishers, Dordrecht.

Musienko A, Tatschl A, Schmidegg K, Kolednik O, Pippan R, Cailletaud G (2007) Threedimensional finite element simulation of a polycrystalline copper specimen. Acta Mater 55: 4121-4136.

Nebozhyn MV, Gilormini P, Ponte Castañeda P (2000) Variational self-consistent estimates for viscoplastic polycrystals with highly anisotropic grains. C R Acad Sci Paris IIb 328: 11-17.

Parton VZ, Buryachenko VA (1990) Stress fluctuations in elastic composites. Sov Phys Dokl 35(2):191-193.

Ponte Castañeda P (1991) The effective mechanical properties of nonlinear isotropic composites. J Mech Phys Solids 39: 45-71.

Ponte Castañeda P (1996) Exact second-order estimates for the effective mechanical properties of nonlinear composites. J Mech Phys Solids 44: 827-862.

Ponte Castañeda P (2002) Second-order homogenization estimates for nonlinear composites incorporating field fluctuations: I- theory. J Mech Phys Solids 50: 737-757.

Raabe D, Sachtleber M, Zhao Z, Roters F, Zaefferer S (2001) Micromechanical and macromechanical effects in grain scale polycrystal plasticity experimentation and simulation. Acta Mater 49: 3433-3441.

Rollett AD, Manohar P (2004) The Monte Carlo method. In: Raabe D, Roters F, Barlat F, Chen LQ (eds) Continuum scale simulations of engineering materials. Wiley, Wenheim, pp.77-111

Sachs G (1928). On the derivation of a condition of flowing. Z Verein Deut Ing 72: 734-736.

Semiatin SL, Bieler TR (2001) The effect of alpha platelet thickness on plastic flow during hot working of Ti-6Al-4V with a transformed microstructure. Acta Mater 49: 3565-3573.

Sulsky D, Zhou SJ, Schreyer HL (1995) Application of a particle-in-cell method to solid mechanics. Comput Phys Commun 87: 236-252.

Taylor GI (1938) Plastic strain in metals. J Inst Met 62: 307-324.

Tomé CN, Canova GR, Kocks UF, Christodoulou N, Jonas JJ (1984) The relation between macroscopic and microscopic strain-hardening in fcc polycrystals. Acta Metall 32: 1637-1653.

Tomé CN, Lebensohn RA (2008). Manual for Code Viscoplastic Self-Consistent (version 7).

Walpole LJ (1969) On the overall elastic moduli of composite materials. J Mech Phys Solids 17: 235-251.

Wang YU, Jin YMM, Khachaturyan AG (2002) Phase field microelasticity theory and modeling of elastically and structurally inhomogeneous solid. J Appl Phys 92: 1351-1360.

Wenk HR, Tomé CN (1999) Modeling dynamic recrystallization of olivine aggregates deformed in simple shear. J Geophys Res B 104: 25513-25527.

Willis JR (1981) Variational and related methods for the overall properties of composites. Adv Appl Mech 21: 1-78.

Winther G, Margulies L, Schmidt S, Poulsen HF (2004) Lattice rotations of individual bulk grains Part II: correlation with initial orientation and model comparison. Acta Mater 52: 2863-2872. 


\section{Appendix: Calculation of Effective Moduli}

\section{Derivatives}

We give here the expressions and algorithm for the calculation of the derivatives of the effective moduli within the context of the VPSC formulation (Lebensohn et al. 2007).

\section{A.1 Calculation of $\partial B_{k j}^{(s)} / \partial M_{u v}^{(r)}$}

From (32), we have [in matrix notation, all indices running from 1 to 5, except the grain indices (r) and (s)]:

$$
\begin{aligned}
\frac{\partial B_{k j}^{(s)}}{\partial M_{u v}^{(r)}}= & -\frac{1}{2}\left[\left(M^{(s)}+\tilde{M}\right)_{k u}^{-1} \delta_{r s} B_{v j}^{(s)}+\left(M^{(s)}+\tilde{M}\right)_{k v}^{-1} \delta_{r s} B_{u j}^{(s)}\right]+ \\
& +\left(M^{(s)}+\tilde{M}\right)^{-1}\left[\frac{\partial \tilde{M}}{\partial M_{u \nu}^{(r)}}\left(I-B^{(s)}\right)+\frac{\partial \tilde{M}}{\partial M_{u v}^{(r)}}\right] .
\end{aligned}
$$

In order not to clutter the notation, the first and second term on the right are written in explicit and implicit index notation, respectively. In the second term, the indices (uv) (i.e., the component of the local compliance with respect to which the derivatives are calculated) appear only to indicate such derivative, while in the first term they appear mixed with the indices that contract. In what follows, we will use this mix of explicit indices and implicit notation, when necessary for the sake of clarity.

Deriving (30), we obtain:

$$
\frac{\partial \tilde{M}_{i j}}{\partial M_{u \nu}^{(r)}}=(I-S)_{i k}^{-1} \frac{\partial S_{k l}}{\partial M_{u \nu}^{(r)}} \psi_{l j}+F_{i p}^{S} \frac{\partial \bar{M}_{p j}}{\partial M_{u \nu}^{(r)}},
$$

where $F^{S}=(I-S)^{-1} S$ and $\psi=F^{S} \tilde{M}+\tilde{M}$. Using the chain rule to express the first derivative on the right, we can write:

$$
\frac{\partial \tilde{M}_{i j}}{\partial M_{u \nu}^{(r)}}=(I-S)_{i k}^{-1} \frac{\partial S_{k l}}{\partial \bar{M}_{p q}} \frac{\partial \bar{M}_{p q}}{\partial M_{u \nu}^{(r)}} \psi_{l j}+F_{i p}^{S} \frac{\partial \bar{M}_{p j}}{\partial M_{u \nu}^{(r)}}=\theta_{i j p q} \frac{\partial \bar{M}_{p q}}{\partial M_{u \nu}^{(r)}},
$$

where

$$
\theta_{i j p q}=(I-S)_{i k}^{-1} \frac{\partial S_{k l}}{\partial \bar{M}_{p q}} \psi_{l j}+F_{i p}^{S} \delta_{j q}
$$


The algorithm for the calculation of $\partial S / \partial \bar{M}$ is given below. Replacing (A3) in (A1) and after some manipulation, we obtain:

$$
\frac{\partial B_{k j}^{(s)}}{\partial M_{u \nu}^{(r)}}=-\frac{1}{2}\left(\chi_{k u}^{(s)} \delta_{r s} B_{v j}^{(s)}+\chi_{k v}^{(s)} \delta_{r s} B_{u j}^{(s)}\right)+\chi^{(s)} \alpha^{(s)}: \frac{\partial \bar{M}}{\partial M_{u \nu}^{(r)}}
$$

where

$$
\begin{gathered}
\chi^{(s)}=\left(M^{(s)}+\tilde{M}\right)^{-1}, \\
\alpha_{i j k l}^{(s)}=\theta_{i m k l}\left(I-B^{(s)}\right)_{m j}+\delta_{i k} \delta_{j l} .
\end{gathered}
$$

\section{A.2 Calculation of $\partial \bar{M}_{i j} / \partial M_{u v}^{(r)}$}

Deriving (44):

$$
\frac{\partial \bar{M}_{i j}}{\partial M_{u \nu}^{(r)}}=\sum_{s} c^{(s)} \delta_{i u} \delta_{k \nu} \delta_{r s} B_{k j}^{(s)}+\sum_{s} c^{(s)} M_{i k}^{(s)} \frac{\partial B_{k j}^{(s)}}{\partial M_{u \nu}^{(r)}} .
$$

Using (A5) and calling $\beta^{(s)}=M^{(s)} \chi^{(s)}$, we get:

$$
\begin{aligned}
\frac{\partial \bar{M}_{i j}}{\partial M_{u \nu}^{(r)}}= & \frac{c^{(r)}}{2}\left(\delta_{i u} B_{v j}^{(r)}+\delta_{i v} B_{u j}^{(r)}\right)-\frac{c^{(r)}}{2}\left(\beta_{i u}^{(r)} B_{v j}^{(r)}+\beta_{i v}^{(r)} B_{u j}^{(r)}\right)+ \\
& \left.+\sum_{s} c^{(s)} \beta^{(s)} \alpha^{(s)}\right) \frac{\partial \bar{M}}{\partial M_{u \nu}^{(r)}}
\end{aligned}
$$

From where:

$$
\Omega_{i j k l} \frac{\partial \bar{M}_{k l}}{\partial M_{u v}^{(r)}}=\pi_{i j}^{(r, u v)}
$$

with

$$
\begin{gathered}
\pi_{i j}^{(r, u v)}=\frac{c^{(r)}}{2}\left[\left(\delta_{i u}-\beta_{i u}^{(r)}\right) B_{v j}^{(r)}+\left(\delta_{i v}-\beta_{i v}^{(r)}\right) B_{u j}^{(r)}\right] . \\
\Omega_{i j k l}=\delta_{i k} \delta_{j l}-\sum_{s} c^{(s)} \beta^{(s)} \alpha^{(s)}
\end{gathered}
$$




\section{A.3 Calculation of $\partial E^{v} / \partial M_{u}{ }^{(r)}{ }_{v}$}

$j$

Deriving (45):

$$
\frac{\partial E_{i}^{o}}{\partial M_{u \nu}^{(r)}}=\sum_{s} c^{(s)} \varepsilon_{k}^{o(s)} \frac{\partial B_{k i}^{(s)}}{\partial M_{u v}^{(r)}} .
$$

Using (A5), we obtain:

$$
\frac{\partial E_{i}^{o}}{\partial M_{u \nu}^{(r)}}=\zeta_{i k l} \frac{\partial \bar{M}_{k l}}{\partial M_{u \nu}^{(r)}}+\kappa_{i}^{(r, u v)},
$$

where

$$
\begin{gathered}
\zeta_{i j k}=\sum_{s} c^{(s)} \varepsilon_{m}^{o(s)} \chi_{m l}^{(s)} \alpha_{l i j k}^{(s)} \\
\kappa_{i}^{(r, u v)}=-\frac{c^{(r)}}{2} \varepsilon_{k}^{o(r)}\left[\chi_{k u}^{(r)} B_{v i}^{(r)}+\chi_{k v}^{(r)} B_{u i}^{(r)}\right]
\end{gathered}
$$

\section{A.4 Calculation of $\partial \bar{G} / \partial M_{u v}^{(r)}$}

Deriving (46):

$$
\frac{\partial \bar{G}}{\partial M_{u \nu}^{(r)}}=\sum_{s} c^{(s)} \varepsilon_{i}^{o(s)} \frac{\partial b_{i}^{(s)}}{\partial M_{u \nu}^{(r)}} .
$$

Deriving (33):

$$
\begin{aligned}
\frac{\partial b_{i}^{(s)}}{\partial M_{u \nu}^{(r)}}= & -\frac{1}{2} \delta_{r s}\left[\chi_{i u}^{(s)} \chi_{\nu l}^{(s)}\left(E_{l}^{o}-\varepsilon_{l}^{o(s)}\right)+\chi_{i \nu}^{(s)} \chi_{u l}^{(s)}\left(E_{l}^{o}-\varepsilon_{l}^{o(s)}\right)\right] \\
& -\chi^{(s)} \frac{\partial \tilde{M}}{\partial M_{u \nu}^{(r)}} \chi^{(s)}\left(E^{o}-\varepsilon^{o(s)}\right)+\chi^{(s)} \frac{\partial E^{o}}{\partial M_{u \nu}^{(r)}} .
\end{aligned}
$$

Replacing (A17) in (A16) and using (A3):

$$
\frac{\partial \bar{G}}{\partial M_{u \nu}^{(r)}}=\varphi_{i j} \frac{\partial M_{i j}^{o}}{\partial M_{u v}^{(r)}}+\vartheta_{i} \frac{\partial E_{i}^{o}}{\partial M_{u \nu}^{(r)}}+\eta^{(r, u v)},
$$


where

$$
\begin{gathered}
\varphi_{i j}=-\left[\sum_{s} c^{(s)} \varepsilon_{k}^{o(s)} \chi_{k l}^{(s)} \chi_{p q}^{(s)}\left(E_{q}^{o}-\varepsilon_{q}^{o(s)}\right)\right] \theta_{l p i j}, \\
\vartheta_{i}=\sum_{s} c^{(s)} \varepsilon_{k}^{o(s)} \chi_{k i}^{(s)}, \\
\eta^{(r, u v)}=-\frac{c^{(r)}}{2} \varepsilon_{i}^{o(r)}\left[\chi_{i u}^{(r)} \chi_{v l}^{(r)}\left(E_{l}^{o}-\varepsilon_{l}^{o(r)}\right)+\chi_{i v}^{(r)} \chi_{u l}^{(r)}\left(E_{l}^{o}-\varepsilon_{l}^{o(r)}\right)\right] .
\end{gathered}
$$

\section{A.5 Calculation of $\partial S / \partial \bar{M}$}

The derivative of Eshelby tensor with respect to the effective compliance appearing in (A4) can be obtained as follows. From (23) and (24), the (symmetric) Eshelby tensor of an ellipsoidal inclusion of radii (a,b,c) embedded in an incompressible ho-

mogenous medium of stiffness $\bar{L}=\bar{M}^{-1}$ is given by (in tensor notation, all indices running from 1 to 3 ):

$$
S_{i j m n}=T_{i j k l}^{s y m} \bar{L}_{k l m n},
$$

where

$$
T_{i j k l}^{s y m}=\frac{a b c}{16 \pi} \int_{0}^{2 \pi} \int_{0}^{\pi} \frac{\lambda_{i j k l}(\alpha)}{[\rho(\alpha)]^{3}} \sin \theta \mathrm{d} \theta \mathrm{d} \varphi,
$$

with

$$
\lambda_{i j k l}=\alpha_{j} \alpha_{l} A_{i k}^{-1}+\alpha_{i} \alpha_{l} A_{j k}^{-1}+\alpha_{j} \alpha_{k} A_{i l}^{-1}+\alpha_{i} \alpha_{k} A_{j l}^{-1},
$$

where the matrix $4 \times 4 \mathrm{~A}$ is given by (A5). In particular:

$$
A_{i k}=\bar{L}_{i j k l} \alpha_{j} \alpha_{l} \quad(i, j=1,3) .
$$

Deriving (A23):

$$
\frac{\partial S_{i j m n}}{\partial \bar{M}}=\frac{\partial T_{i j k l}^{\mathrm{sym}}}{\partial \bar{M}} \bar{L}_{k l m n}+T_{i j k l}^{\mathrm{sym}} \frac{\partial \bar{L}_{k l m n}}{\partial \bar{M}} .
$$

The first derivative on the right is obtained as:

$$
\frac{\partial T_{i j k l}}{\partial \bar{M}}=\frac{a b c}{16 \pi} \int_{0}^{2 \pi} \int_{0}^{\pi} \frac{\partial \lambda_{i j k l}}{\partial \bar{M}} \frac{\sin \theta \mathrm{d} \theta \mathrm{d} \varphi}{[\rho(\alpha)]^{3}} .
$$


Using (A25) and (A26), $\partial \lambda_{i j k l} / \partial \bar{M}$ is calculated as:

$$
\frac{\partial \lambda_{i j k l}}{\partial \bar{M}}=\alpha_{j} \alpha_{l} \frac{\partial A_{i k}^{-1}}{\partial \bar{M}}+\alpha_{i} \alpha_{l} \frac{\partial A_{j k}^{-1}}{\partial \bar{M}}+\alpha_{j} \alpha_{k} \frac{\partial A_{i l}^{-1}}{\partial \bar{M}}+\alpha_{i} \alpha_{k} \frac{\partial A_{j l}^{-1}}{\partial \bar{M}},
$$

where

$$
\frac{\partial A_{i j}^{-1}}{\partial \bar{M}}=-A_{i k}^{-1}\left(\frac{\partial \bar{L}_{k l m n}}{\partial \bar{M}} \alpha_{l} \alpha_{n}\right) A_{m j}^{-1}
$$

The expression $\partial \bar{L}_{k l m n} / \partial \bar{M}$ appearing in (A27) and (A30) is simply the derivative of a tensor with respect to its inverse. In matrix notation (indices running from 1 to 5):

$$
\frac{\partial \bar{L}_{i j}}{\partial \bar{M}_{p q}}=-\frac{1}{2}\left(\bar{L}_{i p} \bar{L}_{q j}+\bar{L}_{i q} \bar{L}_{p j}\right)
$$

